Utah State University

DigitalCommons@USU

Mechanical and Aerospace Engineering Student Mechanical and Aerospace Engineering Student Publications and Presentations

$1-5-2020$

\title{
Minimum Induced Drag for Tapered Wings Including Structural Constraints
}

Jeffrey D. Taylor

Utah State University, jeffdtaylor3891@gmail.com

Doug F. Hunsaker

Utah State University, doug.hunsaker@usu.edu

Follow this and additional works at: https://digitalcommons.usu.edu/mae_stures

Part of the Mechanical Engineering Commons

\section{Recommended Citation}

Taylor, J. D., and Hunsaker, D. F., "Minimum Induced Drag for Tapered Wings Including Structural Constraints," AIAA 2020-2113, AIAA Scitech 2020 Forum, Orlando, Florida, 6-10 January, 2020. (doi:10.2514/6.2020-2113)

This Conference Paper is brought to you for free and open access by the Mechanical and Aerospace Engineering Student Research at DigitalCommons@USU. It has been accepted for inclusion in Mechanical and Aerospace Engineering Student Publications and Presentations by an authorized administrator of DigitalCommons@USU. For more information, please contactdigitalcommons@usu.edu.

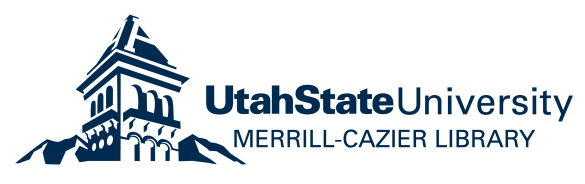




\title{
Minimum Induced Drag for Tapered Wings Including Structural Constraints
}

\author{
Jeffrey D. Taylor* and Douglas F. Hunsaker ${ }^{\dagger}$ \\ Utah State University, Logan, Utah 84322-4130
}

\begin{abstract}
For a wing in steady level flight, the lift distribution that minimizes induced drag depends on a tradeoff between wingspan and wing-structure weight. In 1933, Prandtl suggested that tapered wings have an advantage over rectangular wings due to this tradeoff. However, Prandtl's solutions were obtained using assumptions that correspond to rectangular wings. Therefore, his claim was not analytically proven by his 1933 publication. Here, an approach similar to Prandtl's is taken with more general approximations that apply to wings of arbitrary planform. This more general development is used to study Prandtl's claim about tapered wings. Closed-form solutions for the optimum wingspan and corresponding induced drag are presented for wings having elliptic and linearly-tapered planforms with constraints of fixed wing loading and maximum stress. It is shown that induced drag is minimized with a triangular planform, which gives a reduction in induced drag of up to $24.44 \%$ over the rectangular planform and up to $11.71 \%$ over the elliptic planform. Numerical solutions for the lift distributions that minimize induced drag for each planform are also presented. It is shown that the optimum lift distribution produces up to $5.94 \%$ less induced drag than the elliptic lift distribution when the triangular planform is used.
\end{abstract}

\section{Nomenclature}

$A \quad=$ beam cross-sectional area

$a_{m} \quad=$ fit coefficients in the polynomial approximation of $C_{n}$ for linearly-tapered wings, Eq. (A1)

$B_{n} \quad=$ Fourier coefficients in the lifting-line solution for the dimensionless section-lift distribution, Eq. (1)

$b \quad=$ wingspan

$C_{n} \quad=$ weighting coefficients for $B_{n}$ in the expressions for wing-structure weight, Eqs. (25) and (44)

$C_{\sigma} \quad=$ shape coefficient for the stress-limited design, Eq. (9)

$c \quad=$ local wing section chord length

$\bar{c} \quad=$ wing mean geometric chord, defined as $\bar{c} \equiv S / b$

$c_{r} \quad=$ local wing section chord length at the wing root

$c_{t} \quad=$ local wing section chord length at the wing tip

$D_{i} \quad=$ wing induced drag

$h=$ height of the beam cross-section

$I=$ beam section moment of inertia

$L \quad=$ total wing lift

$\widetilde{L} \quad=$ local wing section lift

$\tilde{M}_{b} \quad=$ local wing section bending moment

$n_{a} \quad=$ load factor, $g$

$n_{g} \quad=$ limiting load factor at the hard-landing design limit

$n_{m} \quad=$ limiting load factor at the maneuvering-flight design limit

$R_{T} \quad$ = wing taper ratio

\footnotetext{
* PhD Candidate, Mechanical and Aerospace Engineering, 4130 Old Main Hill, AIAA Student Member

${ }^{\dagger}$ Assistant Professor, Mechanical and Aerospace Engineering, 4130 Old Main Hill, AIAA Senior Member
} 


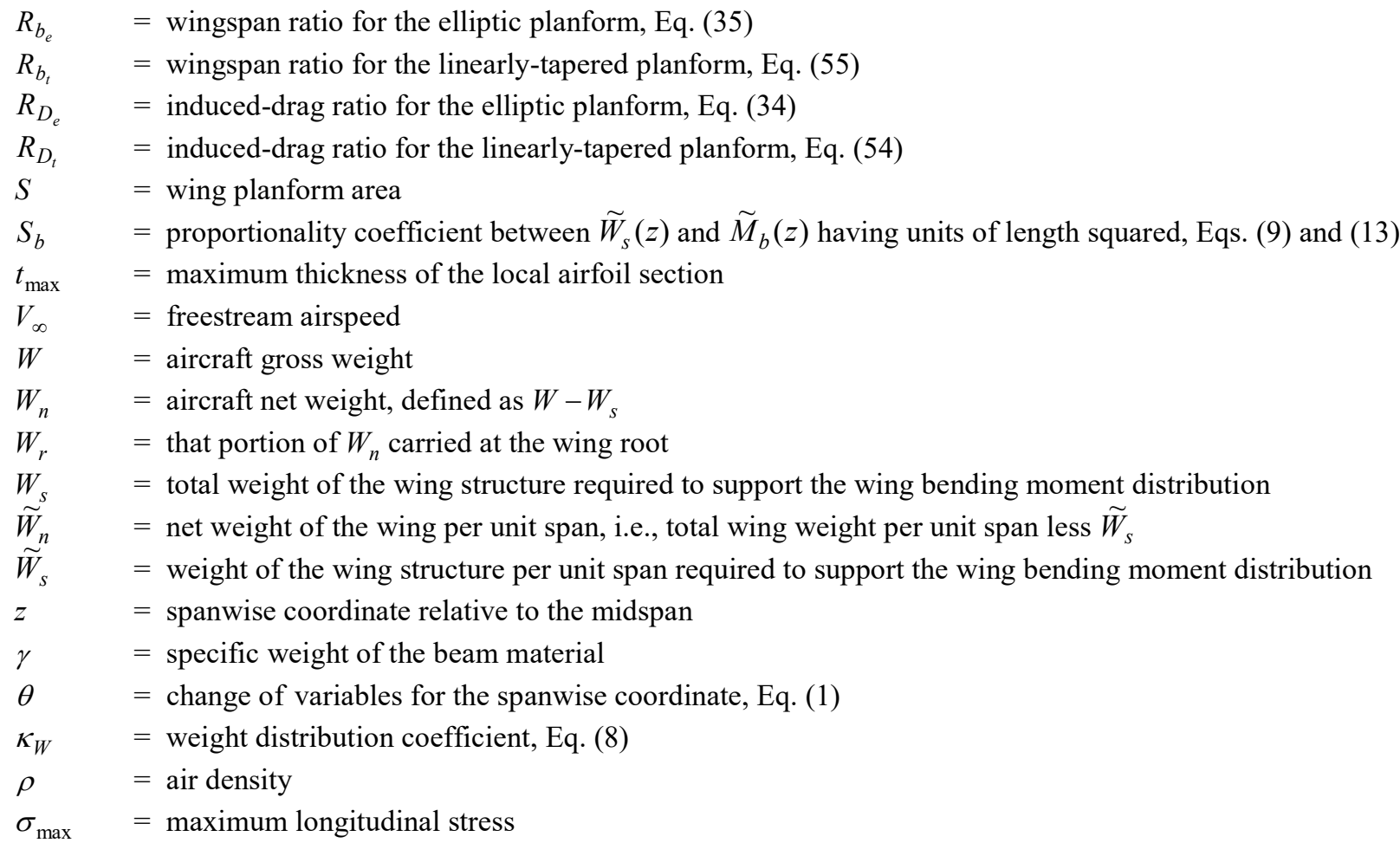

\section{Introduction}

PRANDTL'S classical lifting-line theory [1,2] relates the spanwise lift distribution to the spanwise chord-length and aerodynamic angle-of-attack distributions for an unswept wing immersed in an inviscid, incompressible, uniform flow. If any two of these distributions are known, Prandtl's classical lifting-line equation can be used to find the third. For instance, below stall, any desired lift distribution can be produced on any given planform if the correct twist distribution is used. Given a planform shape, the lifting-line equation can be used to find the twist distribution needed to produce the desired lift distribution [3]. Therefore, in this work, we will treat planform and lift distribution as two independent parameters that are linked through the dependent parameter of wing twist. Lifting-line theory has long been used in the aerospace industry for aerodynamic analyses and optimization, and results based on this theory have been shown to be in good agreement with CFD [4-11]. However, lifting-line theory can also be used to gain insight into the aerodynamic and structural coupling involved in designing a wing for minimum induced drag.

From classical lifting-line theory, the spanwise lift distribution can be written in terms of a Fourier series. Although this series is generally written in an alternate form, here we shall use the dimensionless form [12]

$$
\frac{b \widetilde{L}(\theta)}{L}=\frac{4}{\pi}\left[\sum_{n=1}^{\infty} B_{n} \sin (n \theta)\right] ; \quad \theta \equiv \cos ^{-1}(-2 z / b)
$$

where $B_{1}=1$. Equation (1) can be used to define any dimensionless lift distribution. However, in this paper, we will only consider spanwise-symmetric lift distributions, which have $B_{n}=0$ for all even $n$. In steady level flight, the lift, $L$, is equal to the weight, $W$, and the induced drag can be written in terms of the Fourier coefficients, $B_{n}$, as [12]

$$
D_{i}=\frac{2(W / b)^{2}}{\pi \rho V_{\infty}^{2}}\left(1+\sum_{n=2}^{\infty} n B_{n}^{2}\right)
$$

Equation (2) shows that for a given flight condition, the induced drag is a function of the weight, the wingspan, and the Fourier coefficients that define the lift distribution. For any fixed weight and wingspan, Eq. (2) is minimized by 
using $B_{n}=0$ for all $n \geq 2$. This yields the well-known elliptic lift distribution. Any other lift distribution having nonzero Fourier coefficients incurs a penalty in induced drag.

The elliptic lift distribution can be produced using an untwisted elliptically-tapered planform. Because of this, the elliptically-tapered planform is commonly considered the most efficient planform. The lift distribution produced by any other untwisted planform is non-elliptic and therefore produces more induced drag than the untwisted ellipticallytapered planform at a given span. However, it has been shown that linearly-tapered wings produce nearly elliptic lift distributions at taper ratios near $R_{T}=0.4$ [13] without any twist. Because a wing with elliptic taper is much more difficult to manufacture than a linearly-tapered planform, linearly-tapered planforms with taper ratios near $R_{T}=0.4$ are commonly favored over the elliptic planform to minimize induced drag for a fixed weight and wingspan.

If the weight and wingspan are allowed to vary, the elliptic lift distribution does not necessarily minimize induced drag. Equation (2) shows that the induced drag can be decreased by reducing weight and/or increasing wingspan. However, wingspan cannot be increased arbitrarily because as wingspan increases, the weight of the wing structure, which is proportional to the wing bending moments, also increases. Because of this, if weight and wingspan are not fixed, certain non-elliptic lift distributions that decrease bending moments across the span can allow a larger wingspan than that allowed by the elliptic lift distribution for the same wing-structure weight. Therefore, there exists some optimum wingspan, wing-structure weight, and lift distribution that minimizes induced drag on a wing in steady level flight.

Prandtl seems to be the first to have realized this and published a paper on the topic in 1933 [14]. In that publication, he showed that for a rectangular wing with fixed gross weight and moment of inertia of gross weight, the lift distribution that minimizes induced drag is a bell-shaped lift distribution having $B_{3}=-1 / 3$ and $B_{n}=0$ for all $n \neq 3$. Under Prandtl's design constraints [14], this bell-shaped lift distribution allows a $22.5 \%$ increase in wingspan and an $11.1 \%$ reduction in induced drag over the elliptic lift distribution. In order to obtain analytic results, Prandtl assumed that the wing bending moments are only a function of the lift distribution and that the wing-structure weight makes no contribution to the bending moments. He also assumed that at each section, the bending moment, $\widetilde{M}_{b}(z)$, is related to the wing-structure weight, $\widetilde{W}_{s}(z)$, by a spanwise-invariant proportionality coefficient, $S_{b}[14]$, i.e.,

$$
\widetilde{W}_{s}(z)=\frac{\tilde{M}_{b}(z)}{S_{b}}
$$

In his 1933 paper, Prandtl noted that the lift distribution given by $B_{3}=-1 / 3$ corresponds more closely to that produced by what he called "spitzendigen Flügel" [14] (which can be translated as "pointed-end wings" or "tapered wings") than to the elliptic lift distribution. Within the framework of his solution [14], the elliptic lift distribution performs "noticeably worse" $[14,15]$ than the lift distribution given by $B_{3}=-1 / 3$. Therefore, near the end of his 1933 paper, Prandtl concluded that "tapered wings have an advantage over those with a nearly rectangular profile" $[14,15]$. Prandtl's statement is somewhat vague because any wing having a chord distribution that decreases with span could be considered a tapered wing. For example, elliptically-tapered wings and linearly-tapered wings are two common types of wings that could be considered tapered wings. However, because Prandtl assumed that the proportionality coefficient, $S_{b}$, is constant along the span and did not consider the effects of the chord distribution, his solution applies only to rectangular planforms. Therefore, his claim was not proven by his 1933 results [14]. In fact, it appears that no analytic proof of Prandtl's conclusion, with structural and planform effects included, has ever been shown.

Various analytic or low-order studies on minimizing induced drag using lifting-line theory and similar methods have been published since 1933. Whereas many of these studies approach the problem from a purely aerodynamic point of view [16-24], others follow an approach similar to that taken by Prandtl in 1933 [25-38]. Many of the early analytical studies in this second group consider the wing structure independent of the wing geometry. For instance, Jones [32] used the root bending moment as the primary structural constraint, without considering any physical wing structure. DeYoung [33] replaced the root-bending-moment constraint with a constraint on the bending moment at an arbitrary spanwise location. Later, Jones and Lasinski [34] and Klein and Viswanathan [35,36] incorporated constraints on the integrated bending moment, relating the bending moments to the wing-structure weight using the relationship shown in Eq. (3). However, like Prandtl [14], Jones and Lasinski [34] and Klein and Viswanathan [35,36] did not include the effects of the chord distribution and treated the proportionality coefficient as a fixed parameter, independent of the wing geometry. Löbert [37], on the other hand, introduced a structural constraint based on the ratio of the section bending moment and the wing-section thickness. Because the wing-section thickness is often related to the chord distribution, Löbert's [37] constraint indirectly accounts for the effects of the chord distribution. However, 
instead of investigating how the chord distribution affects the induced drag, Löbert [37] investigated the effect of sweep on the induced drag for a given chord distribution.

More recently, Phillips et al. [12,38] revisited Prandtl's 1933 analysis [14] and relaxed many of his main assumptions, including the assumption that the proportionality coefficient is spanwise invariant and independent of the wing geometry. Instead, Phillips et al. $[12,38]$ related the proportionality coefficient to the local wing dimensions, wing-structure shape, and the wing-structure material. Thus, the development given by Phillips et al. [12] includes the effects of the wing-structure and the chord distribution. However, like Prandtl [14], Phillips et al. [12,38] limited their results to rectangular wings. In this paper, the work of Phillips et al. $[12,38]$ will be extended analytically to identify expressions for the induced drag of non-rectangular wings, including the effects of the chord distribution on the wing structure. The new expressions will then be used to evaluate Prandtl's claim that tapered wings have an advantage over rectangular wings $[14,15]$. As will be shown, the results in this paper demonstrate that Prandtl's claim is indeed correct for elliptically-tapered and linearly-tapered planforms when the effects of planform shape are considered. Because it provides a foundation for the work presented in this paper, a brief review of the work of Phillips et al. $[12,38]$ is given in the following section.

\section{Analytical Foundation}

Whereas Prandtl assumed that the wing bending moments are produced by the lift distribution alone, Phillips et al. [12] assumed that at a given load factor, $n_{a}$, the bending moments are caused by the lift distribution, the wingstructure weight distribution, $\widetilde{W}_{s}(z)$, and the distribution of the net weight of all non-structural components carried in the wing, $\widetilde{W}_{n}(z)$ [12], i.e.,

$$
\tilde{M}_{b}(z)=\int_{z^{\prime}=z}^{b / 2}\left[\widetilde{L}\left(z^{\prime}\right)-n_{a} \widetilde{W}_{n}\left(z^{\prime}\right)-n_{a} \widetilde{W}_{s}\left(z^{\prime}\right)\right]\left(z^{\prime}-z\right) d z^{\prime}, \quad \text { for } z \geq 0
$$

For wing-structure design, the bending moment must be evaluated at the design load factor for maneuvering flight, $n_{m}$, and the design load factor for a hard landing, $n_{g}$. In general, the integral in Eq. (4) must be evaluated numerically. However, it can be evaluated analytically if the weight distribution introduced by Phillips et al. [12] is used, i.e.,

$$
\widetilde{W}_{n}(z)=\left(W-W_{r}\right) \frac{\widetilde{L}(z)}{L}-\widetilde{W}_{s}(z)
$$

where $W_{r}$ is the net weight of all non-structural components carried at the wing root. Equation (5) minimizes the bending moments from Eq. (4) when the weight carried at the root satisfies the condition [12]

$$
W_{r}=\frac{n_{g}-1}{n_{m}+n_{g}} W
$$

Using Eq. (5) in Eq. (4), Phillips et al. [12] found that the bending-moment distribution reduces to a form that is proportional to that used by Prandtl in 1933 [14], i.e.,

$$
\left|\widetilde{M}_{b}(z)\right|=\kappa_{W} W_{r} \int_{z^{\prime}=z}^{b / 2} \frac{\widetilde{L}\left(z^{\prime}\right)}{L}\left(z^{\prime}-z\right) d z^{\prime}, \quad \text { for } z \geq 0
$$

where

$$
\kappa_{W}=\left\{\begin{array}{cl}
n_{m} & W_{r} \geq \frac{n_{g}-1}{n_{m}+n_{g}} W \\
\left(n_{g}-1\right) \frac{W}{W_{r}}-n_{g} & W_{r} \leq \frac{n_{g}-1}{n_{m}+n_{g}} W
\end{array}\right.
$$


Like Prandtl, Phillips et al. [12] also assumed that the bending moments are related to the wing-structure weight by a proportionality coefficient. However, unlike Prandtl, they did not assume that the proportionality coefficient is spanwise invariant for all planforms. Instead, Phillips et al. [12] defined the proportionality coefficient in terms of the local chord, $c(z)$, and the beam properties, i.e.,

$$
W_{s}=2 \int_{z=0}^{b / 2} \frac{\left|\tilde{M}_{b}(z)\right|}{S_{b}(z)} d z ; \quad S_{b}(z) \equiv \frac{C_{\sigma}\left(t_{\max } / c\right) c(z) \sigma_{\max }}{\gamma}, \quad C_{\sigma} \equiv \frac{2 I\left(h / t_{\max }\right)}{A h^{2}}
$$

Although Eq. (9) can be used for any planform shape, in order to obtain analytic results, Phillips et al. [12,38] considered only the rectangular planform, for which $S_{b}$ is constant. Using Eqs. (4) and (5) in Eq. (9), and assuming a rectangular planform with an all-positive, spanwise-symmetric lift distribution, the integral in Eq. (9) can be evaluated to give the total wing-structure weight

$$
W_{s}=\frac{\kappa_{W} W_{r} b^{2}}{32 S_{b}}\left(1+B_{3}\right)
$$

Using the relation given in Eq. (6) and the definition for $S_{b}$ from Eq. (9), Eq. (10) can be solved for the wingspan to give [38]

$$
b=\sqrt[3]{\frac{16 C_{\sigma}\left(t_{\max } / c\right) \sigma_{\max } W_{n}}{\left(1+B_{3}\right) \gamma(W / S)} \frac{n_{m}+n_{g}}{n_{m}\left(n_{g}-1\right)}}
$$

Using Eqs. (10) and (11) in Eq. (2), along with the relation given in Eq. (6), gives the associated induced drag [38]

$$
D_{i}=\frac{9}{2 \pi \rho V_{\infty}^{2}}\left[\frac{\left(1+B_{3}\right) \gamma(W / S) W_{n}^{2}}{16 C_{\sigma}\left(t_{\max } / c\right) \sigma_{\max }} \frac{n_{m}\left(n_{g}-1\right)}{n_{m}+n_{g}}\right]^{2 / 3}\left(1+\sum_{n=2}^{\infty} n B_{n}^{2}\right)
$$

If the wing loading, $W / S$, is fixed, Phillips et al. [38] show that this induced drag is minimized with a lift distribution having $B_{3}=-3 / 8+\sqrt{9 / 64-1 / 12}$ and $B_{n}=0$ for $n=2$ and all $n>3$. This lift distribution, along with Prandtl's 1933 lift distribution and other optimum lift distributions found by Phillips et al. [12,38] under different constraints, differ only in the value of $B_{3}$. All of the optimum lift distributions found by Phillips et al. [12,38] have values for $B_{3}$ that fall somewhere between that of Prandtl's 1933 lift distribution $\left(B_{3}=-1 / 3\right)$ and that of the elliptic lift distribution $\left(B_{3}=0\right)$. However, due to the constraints and assumptions imposed by Prandtl [14] and Phillips et al. [12,38], including that the proportionality coefficient, $S_{b}$, the chord, $c$, and the spar height, $h$, are not a function of $z$, their optimum solutions apply only to wings with a rectangular planform. In this paper, we will relax some of these assumptions and consider wings with non-rectangular planforms.

For non-rectangular wings, including tapered wings, the proportionality coefficient, $S_{b}$, as defined in Eq. (9), is a function of spanwise location. Therefore, for non-rectangular planforms, it is often convenient to rewrite the proportionality coefficient in terms of the mean geometric chord, $\bar{c}=S / b$, such that it remains spanwise invariant, i.e.,

$$
S_{b} \equiv \frac{C_{\sigma}\left(t_{\max } / c\right) \bar{c} \sigma_{\max }}{\gamma}
$$

This new definition for the proportionality coefficient will be used for the remainder of this paper. If Eq. (13) is used to define the proportionality coefficient, Eq. (9) must also be rewritten to give a new expression for the wing-structure weight that includes the effects of the chord distribution

$$
W_{s}=2 \int_{z=0}^{b / 2} \frac{\left|\tilde{M}_{b}(z)\right|}{S_{b} c(z) / \bar{c}} d z
$$


Note that, like Eq. (9), Eqs. (13)-(14) are valid for any arbitrary planform, provided that the planform is expressed using a chord distribution that can be integrated in $z$. However, in light of Prandtl's observations about tapered wings, in this paper, we will consider wings with elliptically-tapered and linearly-tapered planforms. For these planforms, integrating Eq. (14) gives closed-form expressions for the wing-structure weight that can be used to predict the induced drag and identify the lift distributions that minimize induced drag.

\section{Elliptically-Tapered Planforms}

For a wing with fixed wingspan and lift distribution, the elliptically-tapered planform (which will be referred to in this paper as the elliptic planform) is commonly accepted as the most efficient planform because it produces an elliptic lift distribution with no aerodynamic or geometric twist when immersed in a uniform flow. For a wing with an elliptic planform, the normalized chord distribution can be written as

$$
\frac{c(z)}{\bar{c}}=\frac{4}{\pi} \sqrt{1-(2 z / b)^{2}}
$$

where $\bar{c}$ is the mean geometric chord, and is given by

$$
\bar{c}=\frac{2}{b} \int_{z=0}^{b / 2} \sqrt{1-(2 z / b)^{2}} d z=\frac{\pi}{4} c_{r}
$$

Note that the dimensionless chord distribution given in Eq. (15) depends only on the wingspan. Equation (15) can be used in Eq. (14) to give the wing-structure weight required to support the bending moments on a wing with an elliptic planform and any fixed all-positive spanwise-symmetric lift distribution

$$
W_{s}=\frac{\pi}{2 S_{b}} \int_{z=0}^{b / 2} \frac{\left|\tilde{M}_{b}(z)\right|}{\sqrt{1-(2 z / b)^{2}}} d z
$$

where the proportionality coefficient is defined in Eq. (13). If the weight distribution from Eq. (5) is used, the moment distribution from Eq. (7) can be used in Eq. (17) to give

$$
W_{s}=\frac{\pi \kappa_{W} W_{r}}{2 S_{b}} \int_{z=0}^{b / 2} \int_{z^{\prime}=z}^{b / 2} \frac{\widetilde{L}\left(z^{\prime}\right)}{L \sqrt{1-(2 z / b)^{2}}}\left(z^{\prime}-z\right) d z^{\prime} d z
$$

Equation (18) is written in terms of the spanwise coordinate $z$. However, in order to evaluate the integral in Eq. (18), the change of variables from Eq. (1) can be used to rewrite the integrand in a more convenient form. Using the change of variables and the lift distribution from Eq. (1), along with the trigonometric identity $\sin (2 \theta)=2 \cos (\theta) \sin (\theta)$, Eq. (18) becomes

$$
W_{s}=\frac{\kappa_{W} W_{r} b^{2}}{8 S_{b}} \sum_{n=1}^{\infty} B_{n} \int_{\theta=\pi / 2}^{\pi} \int_{\theta^{\prime}=\theta}^{\pi} \sin \left(n \theta^{\prime}\right)\left[\frac{\sin (2 \theta)}{\sin \theta} \sin \theta^{\prime}-\sin \left(2 \theta^{\prime}\right)\right] d \theta^{\prime} d \theta
$$

In this form, the inner integral in Eq. (19) can be evaluated analytically using the relations

$$
\int_{\theta^{\prime}=\theta}^{\pi} \sin \left(n \theta^{\prime}\right) \sin \left(\theta^{\prime}\right) d \theta^{\prime}=\left\{\begin{array}{cc}
\frac{\pi}{2}-\frac{\theta}{2}+\frac{\sin (2 \theta)}{4}, & \text { for } n=1 \\
\frac{\sin [(n+1) \theta]}{2(n+1)}-\frac{\sin [(n-1) \theta]}{2(n-1)}, & \text { for } n \neq 1
\end{array}\right.
$$


and

$$
\int_{\theta^{\prime}=\theta}^{\pi} \sin \left(n \theta^{\prime}\right) \sin \left(2 \theta^{\prime}\right) d \theta^{\prime}=\left\{\begin{array}{cc}
\frac{\pi}{2}-\frac{\theta}{2}+\frac{\sin (4 \theta)}{8}, & \text { for } n=2 \\
\frac{\sin [(n+2) \theta]}{2(n+2)}-\frac{\sin [(n-2) \theta]}{2(n-2)}, & \text { for } n \neq 2
\end{array}\right.
$$

which, when used in Eq. (19), give

$$
\begin{aligned}
W_{s}= & \frac{\kappa_{W} W_{r} b^{2}}{8 S_{b}}\left\{\int_{\theta=\pi / 2}^{\pi} \frac{[2 \pi-2 \theta+\sin (2 \theta)] \sin (2 \theta)}{4 \sin \theta} d \theta-\int_{\theta=\pi / 2}^{\pi} \frac{\sin (3 \theta)-3 \sin \theta}{6} d \theta\right. \\
+ & B_{3} \int_{\theta=\pi / 2}^{\pi} \frac{[\sin (4 \theta)-2 \sin (2 \theta)] \sin (2 \theta)}{8 \sin \theta} d \theta-B_{3} \int_{\theta=\pi / 2}^{\pi} \frac{\sin (5 \theta)-5 \sin \theta}{10} d \theta \\
& +\sum_{n=5}^{\infty} B_{n} \int_{\theta=\pi / 2}^{\pi} \frac{(n-1) \sin [(n+1) \theta]-(n+1) \sin [(n-1) \theta] \sin (2 \theta)}{2\left(n^{2}-1\right) \sin \theta} d \theta \\
& \left.-\sum_{n=5}^{\infty} B_{n} \int_{\theta=\pi / 2}^{\pi} \frac{(n-2) \sin [(n+2) \theta]-(n+2) \sin [(n-2) \theta]}{2\left(n^{2}-4\right)} d \theta\right\}
\end{aligned}
$$

Each of the integrals in Eq. (22) can be evaluated analytically. Carrying out the integration gives a closed-form expression for the wing-structure weight required to support the bending moments on a wing with an elliptic planform, the weight distribution given by Eq. (5), and any all-positive spanwise-symmetric lift distribution

$$
W_{s}=\frac{\kappa_{W} W_{r} b^{2}}{8 S_{b}}\left\{\frac{16}{9}-\frac{\pi}{2}+\sum_{n=3}^{\infty}\left[\frac{16}{n\left(n^{2}-4\right)^{2}}\right] B_{n}\right\}
$$

For convenience, Eq. (23) can be rewritten in the form

$$
W_{s}=\frac{\kappa_{W} W_{r} b^{2}}{8 S_{b}}\left\{C_{1}+\sum_{n=3}^{\infty} C_{n} B_{n}\right\}
$$

where

$$
C_{n} \equiv \begin{cases}\frac{16}{9}-\frac{\pi}{2}, & \text { for } n=1 \\ \frac{16}{n\left(n^{2}-4\right)^{2}}, & \text { for } n \neq 1\end{cases}
$$

Note that whereas the wing-structure weight given in Eq. (10) for a rectangular wing is a function of only the third Fourier coefficient, $B_{3}$, Eq. (24) shows that for the elliptic planform with an all-positive spanwise-symmetric lift distribution, the wing-structure weight is dependent on all odd Fourier coefficients. However, it is also important to note from Eq. (25) that as $n$ increases, the coefficient, $C_{n}$, for each $n$ scales roughly as $1 / n^{5}$, meaning that as $n$ increases, the influence of the Fourier coefficient on the wing-structure weight decreases. 
Because the wing loading, $W / S$, is often fixed by airspeed requirements, it is also sometimes convenient to rewrite Eq. (24) in terms of the wing loading. This can be done by using the relation $\bar{c}=S / b$ and Eq. (13) in Eq. (24) to give

$$
W_{s}=\frac{\gamma(W / S)}{8 C_{\sigma}\left(t_{\max } / c\right) \sigma_{\max }} \frac{\kappa_{W} W_{r} b^{3}}{W}\left(C_{1}+\sum_{n=3}^{\infty} C_{n} B_{n}\right)
$$

Equation (26) can be used to find the weight of the wing structure required for a wing having an elliptic planform with fixed wing loading and fixed gross weight. The corresponding wingspan can be found by rearranging Eq. (26) to give

$$
b=\sqrt[3]{\frac{8 C_{\sigma}\left(t_{\max } / c\right) \sigma_{\max }}{\gamma(W / S)\left(C_{1}+\sum_{n=3}^{\infty} C_{n} B_{n}\right)} \frac{W W_{s}}{\kappa_{W} W_{r}}}
$$

Using Eq. (27) in Eq. (2), the induced drag can be written as

$$
D_{i}=\frac{2}{\pi \rho V_{\infty}^{2}}\left[\frac{\gamma(W / S)\left(C_{1}+\sum_{n=3}^{\infty} C_{n} B_{n}\right)}{8 C_{\sigma}\left(t_{\max } / c\right) \sigma_{\max }} \frac{\kappa_{W} W_{r} W^{2}}{W_{s}}\right]^{2 / 3}\left(1+\sum_{n=3}^{\infty} n B_{n}^{2}\right)
$$

The gross weight, $W$, is the sum of the wing-structure weight, $W_{s}$, and the net weight, $W_{n}$. Equation (28) requires that the gross weight and the wing-structure weight be known. However, in many cases, it is more useful to fix the net weight and allow the gross weight to vary. Applying the relationship given in Eq. (6) to minimize the bending moments, the gross weight can be eliminated from Eq. (26), and the wing-structure weight can be rewritten as

$$
W_{s}=\frac{\gamma(W / S) b^{3}}{8 C_{\sigma}\left(t_{\max } / c\right) \sigma_{\max }} \frac{n_{m}\left(n_{g}-1\right)}{n_{m}+n_{g}}\left(C_{1}+\sum_{n=3}^{\infty} C_{n} B_{n}\right)
$$

Using the relation $W=W_{n}+W_{s}$ and Eq. (29) in Eq. (2), the induced drag can be written as

$$
D_{i}=\frac{2}{\pi \rho V_{\infty}^{2}}\left[\frac{W_{n}}{b}+\frac{\gamma(W / S)\left(C_{1}+\sum_{n=3}^{\infty} C_{n} B_{n}\right)}{8 C_{\sigma}\left(t_{\max } / c\right) \sigma_{\max }} \frac{n_{m}\left(n_{g}-1\right)}{n_{m}+n_{g}} b^{2}\right]^{2}\left(1+\sum_{n=3}^{\infty} n B_{n}^{2}\right)
$$

The wingspan that minimizes the induced drag can be found by differentiating Eq. (30) with respect to the wingspan and setting the result equal to zero. This gives

$$
b=\sqrt[3]{\frac{4 W_{n} C_{\sigma}\left(t_{\max } / c\right) \sigma_{\max }}{\gamma(W / S)\left(C_{1}+\sum_{n=3}^{\infty} C_{n} B_{n}\right)} \frac{\left(n_{m}+n_{g}\right)}{n_{m}\left(n_{g}-1\right)}}
$$

Using Eq. (31) in Eq. (29) gives the wing-structure weight that minimizes the induced drag

$$
W_{s}=\frac{1}{2} W_{n}
$$

Note that Eq. (32) matches the result found by Phillips et al. in [38] for the wing-structure weight that minimizes induced drag for a stress-limited rectangular wing with fixed wing loading. Thus, although Eq. (14) shows that the wing-structure weight is, in general, a function of the chord distribution, the optimum total wing-structure weight, as 
a percent of the net weight, is the same for wings with elliptic planforms and rectangular planforms. Using Eqs. (31) and (32) in Eq. (30) gives the minimum induced drag

$$
D_{i}=\frac{9}{2 \pi \rho V_{\infty}^{2}}\left[\frac{\left(C_{1}+\sum_{n=3}^{\infty} C_{n} B_{n}\right) \gamma(W / S) W_{n}^{2}}{4 C_{\sigma}\left(t_{\max } / c\right) \sigma_{\max }} \frac{n_{m}\left(n_{g}-1\right)}{n_{m}+n_{g}}\right]^{2 / 3}\left(1+\sum_{n=3}^{\infty} n B_{n}^{2}\right)
$$

Because Eq. (33) is in the same form as Eq. (12), the minimum induced drag produced by a wing with an elliptic planform and a given lift distribution can be easily compared to the minimum induced drag produced by a rectangular wing with the same lift distribution by defining an induced-drag ratio

$$
R_{D_{e}} \equiv \frac{\left(D_{i}\right)_{\text {elliptic }}}{\left(D_{i}\right)_{\text {rectangula }}}=\left[\frac{4}{\left(1+B_{3}\right)}\left(C_{1}+\sum_{n=3}^{\infty} C_{n} B_{n}\right)\right]^{2 / 3}
$$

Similarly, comparing Eqs. (31) and (11) gives a wingspan ratio

$$
R_{b_{e}} \equiv \frac{b_{\text {elliptic }}}{b_{\text {rectangular }}}=\sqrt[3]{\frac{1+B_{3}}{4\left(C_{1}+\sum_{n=3}^{\infty} C_{n} B_{n}\right)}}
$$

Because Eq. (34) is a function of an infinite number of Fourier coefficients, in general, minimizing Eq. (34) requires the use of numerical methods. However, Eq. (25) shows that the influence of each Fourier coefficient decreases as $n$ increases. This means that Eqs. (34) and (35) are most heavily influenced by the coefficient $B_{3}$. Therefore, it is useful to consider the case where $B_{n}=0$ for all $n>3$. Figure 1 shows how Eqs. (34) and (35) vary with $B_{3}$ for this special case. Note that results are only shown for $-1 / 3 \leq B_{3} \leq 0$. This is because for $B_{3}<-1 / 3$, the section lift becomes negative near the wingtips. As seen in Eq. (18), the wing-structure weight is proportional to the integral of the lift distribution. Negative lift near the wingtips would result in zero integrated lift and zero wing-structure weight at some inboard location, which is not physically valid. Therefore, in this paper, we assume that the lift distribution is all positive.

Recall that each value of $B_{3}$ in Fig. 1 corresponds to a different lift distribution. For any given planform, any lift distribution can be obtained using wing twist. The elliptic planform is commonly considered the most efficient planform because it produces the elliptic lift distribution with no twist. However, Fig. 1 shows that when structural effects are included, the elliptic planform produces less induced drag than the rectangular planform not only for the elliptic lift distribution, but for all of the lift distributions shown in Fig. 1. The induced-drag ratio is minimized, and the wingspan ratio is maximized, using $B_{3}=-1 / 3$, which corresponds to Prandtl's 1933 lift distribution. This supports Prandtl's claim that "tapered wings have an advantage over those with a nearly rectangular profile" $[14,15]$,

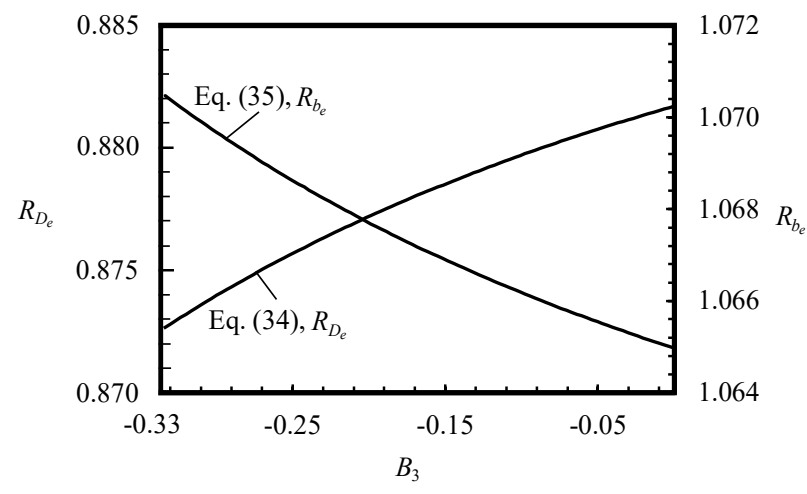

Fig. 1 Ratio of the induced drag and corresponding wingspan produced by an elliptic planform to those produced by a rectangular planform, plotted as a function of $B_{3}$. 
especially when Prandtl's 1933 lift distribution is used. Using this lift distribution, Eqs. (34) and (35) show that the elliptic planform produces up to $12.73 \%$ less induced drag than the rectangular planform with a wingspan increase of $7.05 \%$. However, although the induced-drag ratio in Eq. (34) is minimized using Prandtl's 1933 lift distribution, this lift distribution is not the same as the lift distribution that minimizes the induced drag given in Eq. (33). As will be shown later, the optimum lift distribution that gives an absolute minimum in induced drag for the elliptic planform has a $B_{3}$ value that falls somewhere between that of the elliptic lift distribution and that of Prandtl's 1933 lift distribution.

\section{Linearly-Tapered Planforms}

The process that was shown in the previous section for elliptic planforms can be repeated for wings with linearlytapered planforms. Linearly-tapered wings are commonly used on modern aircraft as a tradeoff between efficiency and ease of manufacture. Consider a linearly-tapered wing with tip chord $c_{t}$ and root chord $c_{r}$. The wing taper ratio is defined as $R_{T} \equiv c_{t} / c_{r}$, and the normalized chord distribution can be written in terms of the taper ratio as

$$
\frac{c(z)}{\bar{c}}=\frac{2\left[1-\left(1-R_{T}\right)|2 z / b|\right]}{1+R_{T}}
$$

where $\bar{c}$ is simply the average of the root chord and the tip chord, and can be expressed in terms of the root chord and the taper ratio as

$$
\bar{c}=\frac{c_{r}}{2}\left(1+R_{T}\right)
$$

Using Eq. (36) in Eq. (14), along with the definition of $S_{b}$ given in Eq. (13), the wing-structure weight required to support the bending moments on a wing with a linearly-tapered planform becomes

$$
W_{s}=\frac{\left(1+R_{T}\right)}{S_{b}} \int_{z=0}^{b / 2} \frac{\left|\tilde{M}_{b}(z)\right|}{1-\left(1-R_{T}\right)|2 z / b|} d z
$$

If the weight distribution given by Eq. (5) is used, the bending-moment distribution given by Eq. (7) can be used in Eq. (38) to give

$$
W_{s}=\frac{\kappa_{W} W_{r}\left(1+R_{T}\right)}{S_{b}} \int_{z=\pi / 2}^{b / 2} \int_{z^{\prime}=z}^{b / 2} \frac{\widetilde{L}\left(z^{\prime}\right)}{L\left[1-\left(1-R_{T}\right)|2 z / b|\right]}\left(z^{\prime}-z\right) d z^{\prime} d z
$$

For convenience, Eq. (39) can be rewritten in a form similar to Eq. (19) using the lift distribution and change of variables from Eq. (1). This gives

$$
W_{s}=\frac{\kappa_{W} W_{r} b^{2}\left(1+R_{T}\right)}{4 \pi S_{b}} \sum_{n=0}^{\infty} B_{n} \int_{\theta=\pi / 2}^{\pi} \int_{\theta^{\prime}=\theta}^{\pi} \frac{\sin \left(n \theta^{\prime}\right)\left[\sin (2 \theta) \sin \theta^{\prime}-\sin \left(2 \theta^{\prime}\right) \sin \theta\right]}{1+\left(1-R_{T}\right) \cos \theta} d \theta^{\prime} d \theta
$$

Using the integral identities given in Eqs. (20) and (21), the inner integral from Eq. (40) can be evaluated analytically. For any spanwise-symmetric lift distribution, the result of these evaluations can be written as 


$$
\begin{aligned}
W_{s}= & \frac{\kappa_{W} W_{r} b^{2}\left(1+R_{T}\right)}{4 \pi S_{b}}\left\{\int_{\theta=\pi / 2}^{\pi} \frac{[2 \pi-2 \theta+\sin (2 \theta)] \sin (2 \theta)}{4\left[1+\left(1-R_{T}\right) \cos \theta\right]} d \theta-\int_{\theta=\pi / 2}^{\pi} \frac{[\sin (3 \theta)-3 \sin \theta] \sin \theta}{6\left[1+\left(1-R_{T}\right) \cos \theta\right]} d \theta\right. \\
& +B_{3} \int_{\theta=\pi / 2}^{\pi} \frac{[\sin (4 \theta)-2 \sin (2 \theta)] \sin (2 \theta)}{8\left[1+\left(1-R_{T}\right) \cos \theta\right]} d \theta-B_{3} \int_{\theta=\pi / 2}^{\pi} \frac{[\sin (5 \theta)-5 \sin \theta] \sin \theta}{10\left[1+\left(1-R_{T}\right) \cos \theta\right]} d \theta \\
& +B_{n} \int_{\theta=\pi / 2}^{\pi} \frac{(n-1) \sin [(n+1) \theta]-(n+1) \sin [(n-1) \theta]}{2\left(n^{2}-1\right)\left[1+\left(1-R_{T}\right) \cos \theta\right]} \sin (2 \theta) d \theta \\
& \left.-B_{n} \int_{\theta=\pi / 2}^{\pi} \frac{(n-2) \sin [(n+2) \theta]-(n+2) \sin [(n-2) \theta]}{2\left(n^{2}-4\right)\left[1+\left(1-R_{T}\right) \cos \theta\right]} \sin \theta d \theta\right\}
\end{aligned}
$$

In general, the complexity of the integrals in Eq. (41) prohibits any simple analytical evaluation. However, for the specific case of a rectangular wing $\left(R_{T}=1\right)$, the integrals can be evaluated analytically, and Eq. (41) reduces to

$$
W_{s}=\frac{\kappa_{W} W_{r} b^{2}}{2 \pi S_{b}}\left(\frac{\pi}{16}+\frac{\pi}{16} B_{3}\right)
$$

which is equivalent to Eq. (10). Note that for a rectangular wing, $\bar{c}=c(z)$, and the definition of $S_{b}$ given in Eq. (13) is the same as that given in Eq. (9).

Here, again, it is convenient to rewrite Eq. (41) in terms of the coefficients $C_{n}$

$$
W_{s}=\frac{\kappa_{W} W_{r} b^{2}\left(1+R_{T}\right)}{4 \pi S_{b}}\left(C_{1}+\sum_{n=3}^{\infty} C_{n} B_{n}\right)
$$

where

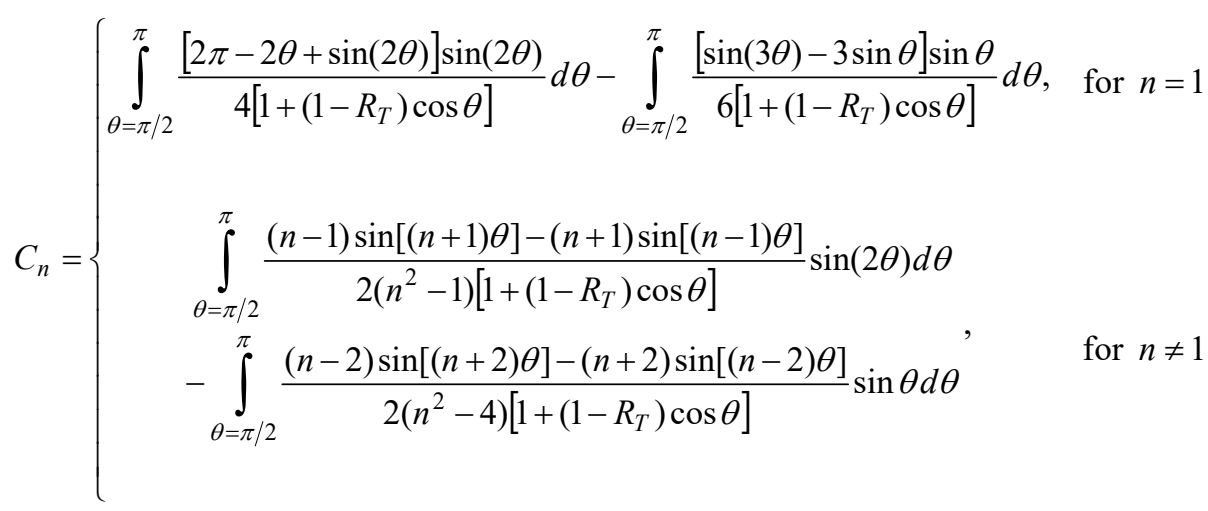

Note that whereas the coefficients, $C_{n}$, for the elliptic planform can be found analytically, the coefficients given in Eq. (44) must be found numerically. This can be done using any high-order integration scheme. Because numerical integration techniques require that the integrand be evaluated at the limits of integration, special care must be taken in the case of a wing with a triangular planform $\left(R_{T}=0\right)$. In this case, each of the integrands in Eq. (44) is indeterminate when evaluated at $\theta=\pi$. Thus, L'hospital's rule can be used to evaluate the limit of each integrand as $\theta$ approaches $\pi$. Applying L'hospital's rule twice to each of the integrands in Eq. (44) gives 


$$
\lim _{\theta \rightarrow \pi} C_{n}=0
$$

Using Simpson's rule and a step size of $d \theta=1.5 \times 10^{-4}$, solutions to Eq. (44) were obtained for taper ratios in the range $0 \leq R_{T} \leq 1$. The resulting values of $C_{n}$ for all odd $n$ in the range $1 \leq n \leq 29$ are given in the appendix, along with closed-form expressions that can be used to approximate $C_{n}$ as a function of taper ratio in the range $0.2 \leq R_{T} \leq 1.5$.

If the wing loading is fixed, Eq. (43) can be rewritten in terms of the gross weight using Eq. (13) and the relation $\bar{c}=S / b$ to give

$$
W_{s}=\frac{\gamma(W / S)\left(1+R_{T}\right)}{4 \pi C_{\sigma}\left(t_{\max } / c\right) \sigma_{\max }} \frac{\kappa_{W} W_{r} b^{3}}{W}\left(C_{1}+\sum_{n=3}^{\infty} C_{n} B_{n}\right)
$$

Rearranging Eq. (46) gives the wingspan allowed by a given lift distribution and wing-structure weight on a wing with a linearly-tapered planform with fixed weight and wing loading

$$
b=\sqrt[3]{\frac{4 \pi C_{\sigma}\left(t_{\max } / c\right) \sigma_{\max }}{\gamma\left(1+R_{T}\right)(W / S)\left(1+\sum_{n=3}^{\infty} C_{n} B_{n}\right)} \frac{W W_{s}}{\kappa_{W} W_{r}}}
$$

Using Eq. (47) in Eq. (2) gives the induced drag

$$
D_{i}=\frac{2}{\pi \rho V_{\infty}^{2}}\left[\frac{\left(1+R_{T}\right)(W / S)\left(C_{1}+\sum_{n=3}^{\infty} C_{n} B_{n}\right) \gamma}{4 \pi C_{\sigma}\left(t_{\max } / c\right) \sigma_{\max }} \frac{\kappa_{W} W_{r} W^{2}}{W_{s}}\right]^{2 / 3}\left(1+\sum_{n=3}^{\infty} n B_{n}^{2}\right)
$$

If the gross weight is not known, the weight constraint from Eq. (6) can be used in Eq. (46) to give

$$
W_{s}=\frac{\gamma(W / S)\left(1+R_{T}\right) b^{3}}{4 \pi C_{\sigma}\left(t_{\max } / c\right) \sigma_{\max }} \frac{n_{m}\left(n_{g}-1\right)}{n_{m}+n_{g}}\left(C_{1}+\sum_{n=3}^{\infty} C_{n} B_{n}\right)
$$

Using the relation $W=W_{s}+W_{n}$ and Eq. (49) in Eq. (2) gives the induced drag

$$
D_{i}=\frac{2}{\pi \rho V_{\infty}^{2}}\left[\frac{W_{n}}{b}+\frac{\gamma(W / S)\left(1+R_{T}\right)\left(C_{1}+\sum_{n=3}^{\infty} C_{n} B_{n}\right)}{4 \pi C_{\sigma}\left(t_{\max } / c\right) \sigma_{\max }} \frac{n_{m}\left(n_{g}-1\right)}{n_{m}+n_{g}} b^{2}\right]^{2}\left(1+\sum_{n=3}^{\infty} n B_{n}^{2}\right)
$$

The wingspan that minimizes Eq. (50) is

$$
b=\sqrt[3]{\frac{2 \pi W_{n} C_{\sigma}\left(t_{\max } / c\right) \sigma_{\max }}{\left(1+R_{T}\right) \gamma(W / S)\left(C_{1}+\sum_{n=3}^{\infty} C_{n} B_{n}\right)} \frac{\left(n_{m}+n_{g}\right)}{n_{m}\left(n_{g}-1\right)}}
$$

When used in Eq. (49), this wingspan gives the wing-structure weight that minimizes induced drag for a linearlytapered wing with fixed wing loading

$$
W_{s}=\frac{1}{2} W_{n}
$$


which is the same result as that shown in Eq. (32). Thus we see that for the planforms considered in this paper, the optimum wing-structure weight is always $W_{n} / 2$, independent of the planform shape. Using Eq. (51) in Eq. (50) gives the minimum induced drag

$$
D_{i}=\frac{9}{2 \pi \rho V_{\infty}^{2}}\left[\frac{\left(1+R_{T}\right)\left(C_{1}+\sum_{n=3}^{\infty} C_{n} B_{n}\right) \gamma(W / S) W_{n}^{2}}{2 \pi C_{\sigma}\left(t_{\max } / c\right) \sigma_{\max }} \frac{n_{m}\left(n_{g}-1\right)}{n_{m}+n_{g}}\right]^{2 / 3}\left(1+\sum_{n=3}^{\infty} n B_{n}^{2}\right)
$$

Note that for a rectangular wing, Eq. (53) reduces to the same result given in Eq. (12) for the stress-limited design of a rectangular wing with fixed wing loading and fixed net weight. However, to compare the minimum induced drag produced by linearly-tapered planforms having $R_{T} \neq 1$ to that produced by the rectangular planform, it is convenient to define an induced-drag ratio for linearly-tapered wings, i.e.,

$$
R_{D_{t}} \equiv \frac{\left(D_{i}\right)_{\text {tapered }}}{\left(D_{i}\right)_{\text {rectangular }}}=\left[\frac{8\left(1+R_{T}\right)}{\pi\left(1+B_{3}\right)}\left(C_{1}+\sum_{n=3}^{\infty} C_{n} B_{n}\right)\right]^{2 / 3}
$$

and a wingspan ratio for linearly-tapered wings, i.e.,

$$
R_{b_{t}} \equiv \frac{b_{\text {tapered }}}{b_{\text {rectangula }}}=\left[\frac{\pi\left(1+B_{3}\right)}{8\left(1+R_{T}\right)\left(C_{1}+\sum_{n=3}^{\infty} C_{n} B_{n}\right)}\right]^{1 / 3}
$$

Note that when the values for $C_{n}$ and $R_{T}$ for a rectangular wing are used in Eqs. (54) and (55), the induced-drag ratio and the wingspan ratio reduce to $R_{b_{t}}=R_{D_{t}}=1$. Fully minimizing Eq. (54) requires the use of numerical methods. However, the solutions to Eq. (44) show that, like Eq. (34), Eq. (54) is most heavily influenced by $B_{3}$. Therefore, we again consider the case where $B_{n}=0$ for all $n>3$. For this special case, the variation in Eq. (54) with $B_{3}$ is shown in Fig. 2 for several linearly-tapered planforms having $0 \leq R_{T} \leq 1$. The results from Eq. (34) are also included for reference. The variation in Eq. (55) with $B_{3}$ is shown in Fig. 3 for the same range of taper ratios, along with results from Eq. (35). Again, we assume that the lift distribution is all positive. Therefore, results are only shown for $-1 / 3 \leq B_{3} \leq 0$.

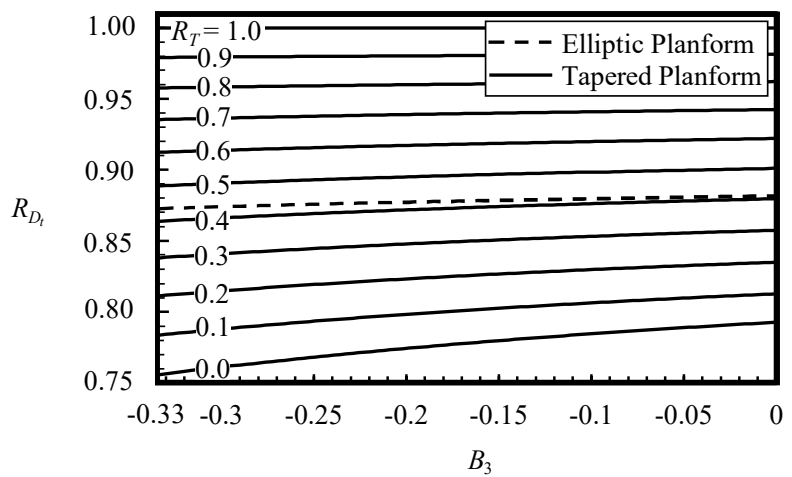

Fig. 2 Ratio of the induced drag produced by the elliptic planform and linearly-tapered planforms with $0 \leq R_{T} \leq 1$ to that produced by a rectangular planform, plotted as a function of $B_{3}$. 


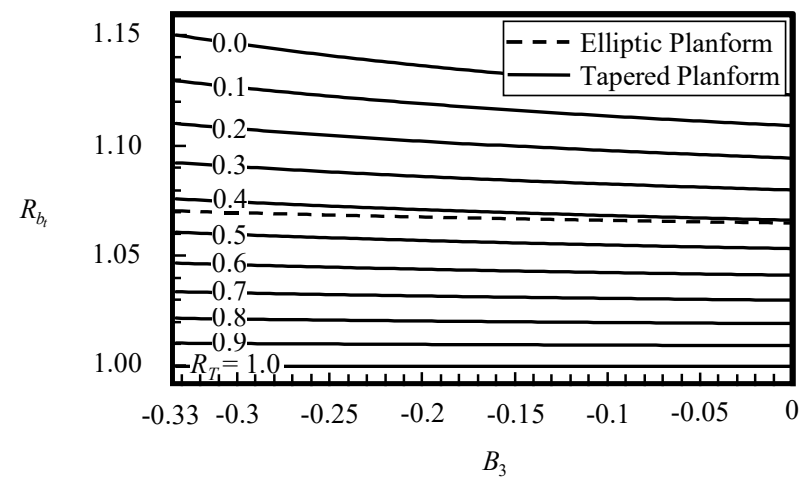

Fig. 3 Ratio of the allowable wingspan for the elliptic planform and linearly-tapered planforms with $0 \leq R_{T} \leq 1$ to the allowable wingspan for rectangular planform, plotted as a function of $B_{3}$.

As was true for the elliptic planform, the lift distribution that minimizes the induced-drag ratio and maximizes the wingspan ratio for all of the linearly-tapered planforms shown in Figs. 2 and 3 is Prandtl's 1933 lift distribution $\left(B_{3}=-1 / 3\right)$. However, it is interesting to note that as taper ratio decreases, the degree to which $B_{3}$ influences the induced-drag ratio and wingspan ratio increases. Therefore, for this case, the induced-drag ratio is minimized, and the wingspan ratio is maximized, using a triangular wing with $R_{T}=0$. When compared to the elliptic planform, this planform produces up to $11.71 \%$ less induced drag. Equations (54) and (55) show that when compared to the rectangular planform, the triangular planform can reduce induced drag by up to $24.44 \%$, with a wingspan increase of $15.04 \%$. Thus, Prandtl's argument that "tapered wings have an advantage over those with a nearly rectangular profile" $[14,15]$ holds when the effects of taper on wing-structure weight are taken into account for all of the lift distributions considered here, with the greatest advantage at $B_{3}=-1 / 3$. However, the reader is reminded that although the lift distribution having $B_{3}=-1 / 3$ minimizes Eq. (54), it may not be the lift distribution that gives an absolute minimum in induced drag for the linearly-tapered planform. This lift distribution is discussed further in the following section.

\section{Optimum Lift Distributions}

Equations (28) and (48) give the induced drag for a wing with fixed gross weight and wing-structure weight, and Eqs. (33) and (53) give the induced drag for a wing with fixed net weight. However, it is important to note that Eqs. (33) and (53) were obtained under the assumption that the wing-structure weight satisfies Eqs. (32) and (52). Thus, because gross weight is the sum of the wing-structure weight and the net weight, minimizing induced drag under the constraint of fixed net weight is, in effect, the same as minimizing induced drag for a wing with fixed gross weight and the wing-structure weight set at one-half the net weight. Therefore, in this section, we will only consider the constraint of fixed net weight.

Under this constraint, Eqs. (33) and (53) give the minimum induced drag for wings with elliptic and linearlytapered planforms, respectively, given a known lift distribution and fixed wing loading. However, neither equation produces an absolute minimum in induced drag unless the optimum lift distribution is also used. Because the wingstructure weight and induced drag are both functions of all the Fourier coefficients that define the lift distribution, in general, the optimum lift distribution is also a function of all the Fourier coefficients. Therefore, to find the lift distribution that minimizes induced drag, the Fourier series must be truncated at a finite value of $n$, and a numerical optimization framework must be employed. For example, Fig. 4 shows the lift distributions that minimize Eqs. (33) and (53) for the elliptic planform and for linearly-tapered planforms with $0 \leq R_{T} \leq 1$. Each lift distribution was found using the Broyden-Fletcher-Goldfarb-Shanno (BFGS) [40-43] method with the Fourier coefficients, $B_{n}$, for all odd $n$ in the range $1 \leq n \leq 29$. The elliptic lift distribution, Prandtl's 1933 lift distribution [14], and the lift distribution produced by an untwisted linearly-tapered wing with aspect ratio 8 and $R_{T}=0$ are also included for reference in Fig. 4. The values of $B_{n}$ that correspond to the optimum lift distributions for wings having the elliptic planform and linearly-tapered planforms with $0 \leq R_{T} \leq 1$ are given in the appendix. Note that because we only wish to consider spanwise-symmetric lift distributions, each even Fourier coefficient is identically zero. 


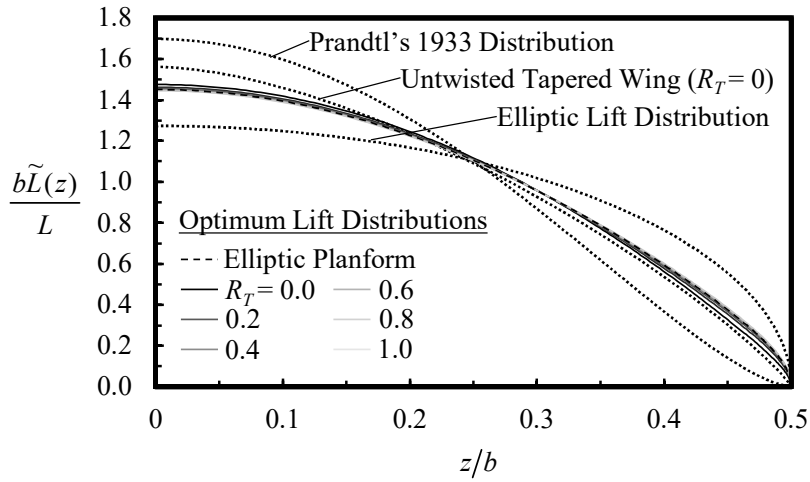

Fig. 4 Solutions for the lift distributions that minimize induced drag for an elliptic planform and linearlytapered planforms with $0 \leq R_{T} \leq 1$.

Figure 4 shows that for each of the planforms considered here, including the elliptic planform, the optimum lift distribution takes neither a bell shape nor an elliptic shape. Instead, each of the optimum lift distributions takes a shape that is somewhat similar to the lift distribution produced by an untwisted linearly-tapered wing with $R_{T}=0$. This agrees with Prandtl's observation that the lift distribution that minimizes induced drag is not elliptic but corresponds more closely to that produced by tapered wings [14,15]. It is also interesting to note that for the planforms considered here, the optimum lift distribution is only a weak function of planform shape.

The optimum lift distributions shown in Fig. 4 were obtained by truncating the Fourier series in Eq. (1) at $n=29$. Because the lift distributions that truly minimize Eqs. (33) and (53) are a function of an infinite number of Fourier coefficients, the results shown in Fig. 4 are only an approximation. However, as seen in Eqs. (25) and (44), as $n$ increases, the magnitude of the coefficients, $C_{n}$, for wings with elliptic and linearly-tapered planforms decrease. This means that the relative influence of each Fourier coefficient on the wing-structure weight, wingspan, and induced drag also decreases as $n$ increases. An example of this is given in Fig. 5, which shows the percent change in induced drag caused by including Fourier coefficients up to $n=29$ in the definition for the optimum lift distribution. The percent change in induced drag shown in Fig. 5 at point $n$ is the percent change between the induced drag obtained using $n$ Fourier coefficients and the induced drag obtained by including up to $n-2$ Fourier coefficients. For example, for the data point at $n=7$, the percent change in induced drag is the percent change between the induced drag obtained by including coefficients up to $n=7$ in the optimum lift distribution and the induced drag obtained by including coefficients up to $n=5$ in the optimum lift distribution.

Note that as $n$ increases, the effect of the corresponding Fourier coefficient on the induced drag decreases, as expected. With as few as two Fourier coefficients $(n=5)$, the percent change in induced drag drops below $0.1 \%$ for all the planforms shown. This suggests that lift distributions at or near the optimum lift distribution are dominated by $B_{3}$ and can be described using $B_{3}$ alone with little loss in accuracy. Figure 6 shows the value of $B_{3}$ in the optimum lift distribution for the elliptic planform and linearly-tapered planforms with $0 \leq R_{T} \leq 1$. Note that all of the $B_{3}$ values shown in Fig. 6 fall between that of the elliptic lift distribution $\left(B_{3}=0\right)$ and that of Prandtl's 1933 lift distribution $\left(B_{3}=-1 / 3\right)$.

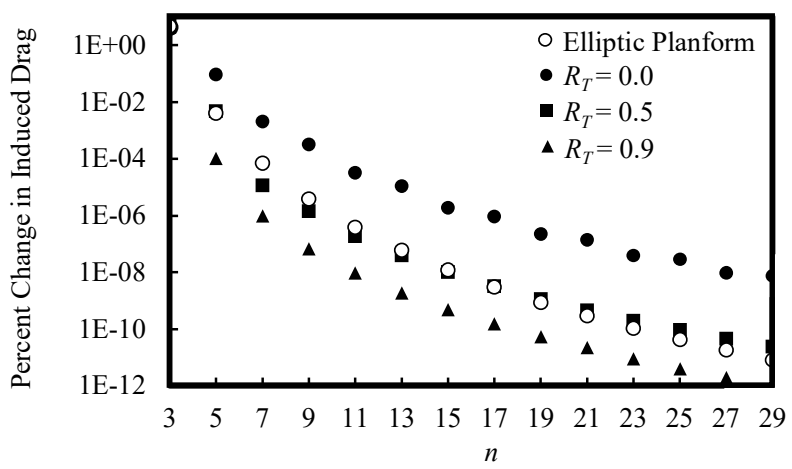

Fig. 5 Percent change in minimum induced drag resulting from including up to $n$ Fourier coefficients in the solution for the optimum lift distribution. 


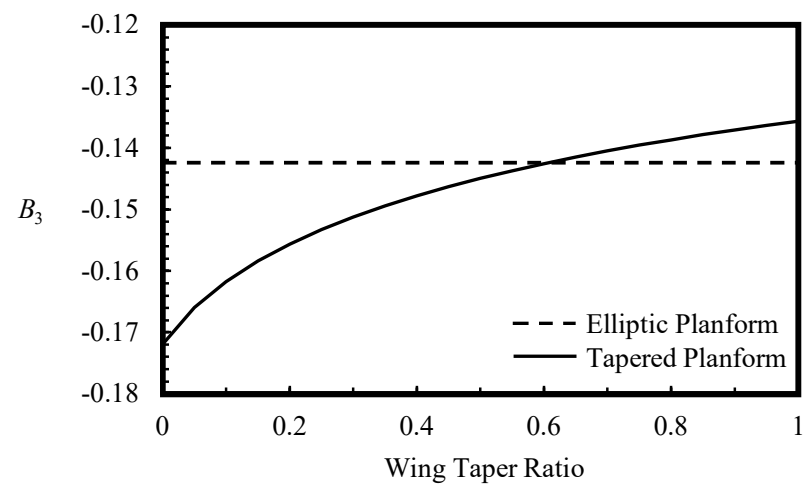

Fig. $6 B_{3}$ values in the Fourier series defining the optimum lift distributions for the elliptic planform and linearly-tapered planforms with $0 \leq R_{T} \leq 1$.

Although the optimum lift distributions shown in Fig. 4 are all very similar in shape, Fig. 6 shows that the value of $B_{3}$ for each lift distribution exhibits a well-defined trend. As taper ratio decreases, minimum induced drag is obtained with lower values of $B_{3}$. In general, as the value of $B_{3}$ decreases, the lift distribution is shifted more toward the root, which means that at low taper ratios, slightly more lift is shifted inboard than at taper ratios near $R_{T}=1$.

For an elliptic planform, the optimum wingspan and minimum possible induced drag are obtained using Eqs. (31) and (33) with the optimum lift distribution for the elliptic planform. For linearly-tapered planforms, the optimum wingspan and the minimum possible induced drag are obtained using Eqs. (51) and (53) with the optimum lift distribution for the linearly-tapered planform. When these values are compared to the wingspan and induced drag obtained using the elliptic lift distribution, the percent change in wingspan and induced drag depends on the planform shape. Figures 7 and 8 show the percent change in induced drag and wingspan, respectively, obtained using the optimum lift distribution compared to those obtained using a fixed elliptic lift distribution for the elliptic planform and linearly-tapered planforms with $0 \leq R_{T} \leq 1$.

The trends shown in Figs. 7 and 8 reveal that the effect of using the optimum lift distribution instead of the elliptic lift distribution on the minimum induced drag and corresponding wingspan is greater for wings with low taper ratios than for wings with nearly rectangular planforms. The most significant changes in induced drag and corresponding wingspan occur at a taper ratio of $R_{T}=0$. At this taper ratio, the optimum lift distribution allows a $7.63 \%$ increase in wingspan and a 5.94\% reduction in induced drag over the elliptic lift distribution. Thus, it is shown that Prandtl's argument for tapered wings $[\mathbf{1 4 , 1 5}]$ holds not only when the lift distribution is fixed, but also when the lift distribution is optimized for each planform.

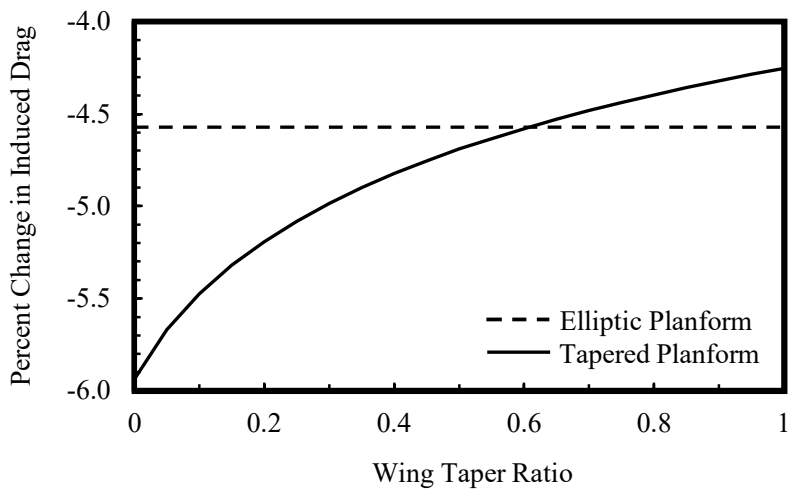

Fig. 7 Percent change in minimum induced drag produced by the optimum lift distribution compared to the elliptic lift distribution for the elliptic planform and linearly-tapered planforms with $0 \leq R_{T} \leq 1$. 


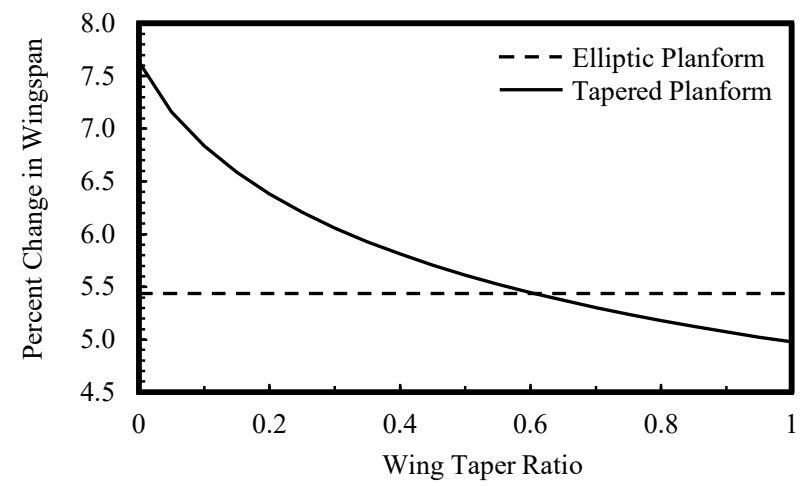

Fig. 8 Percent change in wingspan allowed by the optimum lift distribution compared to the elliptic lift distribution for the elliptic planform and linearly-tapered planforms with $0 \leq R_{T} \leq 1$.

For all of the results shown here, it is important to remember that the aspect ratio is not fixed. Because wing loading and gross weight are constant, the wing area must also be constant. At lower taper ratios, Eqs. (31) and (51) predict higher wingspans and, therefore, higher aspect ratios than at high taper ratios. Several examples of high-aspectratio, low-taper-ratio wings can be found in nature, particularly on high-endurance birds, such as the wandering albatross. Therefore, it should not be surprising that the results shown here predict that minimum induced drag is obtained using a high-aspect-ratio, low-taper-ratio wing when structural constraints are considered.

\section{Example Results}

As an example of minimizing induced drag for an elliptic or linearly-tapered planform, consider an aircraft with net weight fixed at $W_{n}=7000 \mathrm{lbf}$, wing loading fixed at $W / S=30$, and the additional parameters $C_{\sigma}=0.165$, $n_{m}=n_{g}=3.75, t_{\max } / c=0.12, \sigma_{\max }=15 \times 10^{3} \mathrm{psi}, \gamma=0.10 \mathrm{lbf} / \mathrm{in}^{3}, V_{\infty}=200 \mathrm{ft} / \mathrm{s}$, and $\rho=0.0023769 \mathrm{slug} / \mathrm{ft}^{3}$. The weight distributions given in Eqs. (5) and (6) are used to minimize the critical wing bending moments.

Minimum induced drag is obtained by using the optimum lift distributions from Fig. 4 in Eq. (33) for the elliptic planform and Eq. (53) for the linearly-tapered planform. Minimum induced-drag solutions for each of these cases are shown in black in Fig. 9. The wingspans that correspond to each of these cases are found by using the optimum lift distributions from Fig. 4 in Eqs. (31) and (51), and are shown in black in Fig. 10. For reference, the induced drag and corresponding allowable wingspan for the elliptic planform and linearly-tapered planform with a fixed elliptic lift distribution are also included in gray in Figs. 9 and 10.

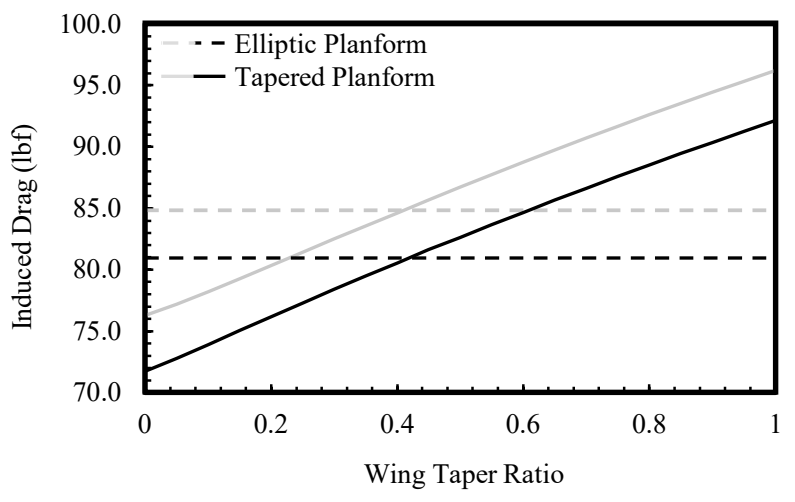

Fig. 9 Example minimum-induced-drag solutions for the elliptic planform and linearly-tapered planforms with $0 \leq R_{T} \leq 1$. Black: Optimum Lift Distribution, Gray: Elliptic Lift Distribution. 


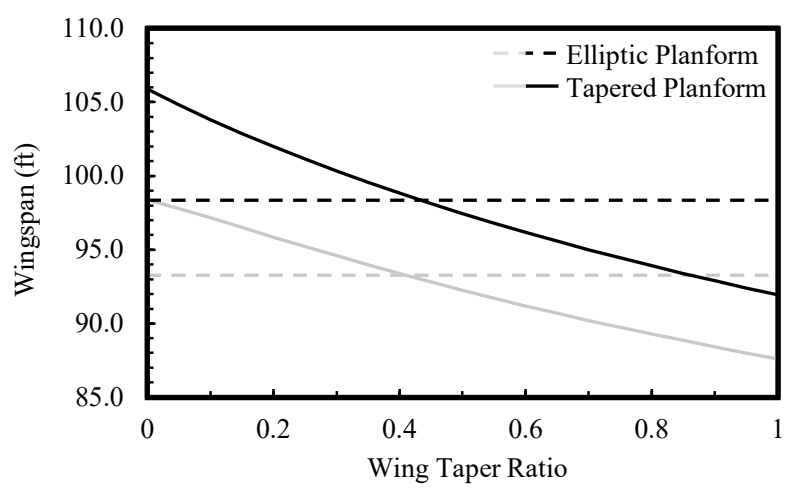

Fig. 10 Example wingspan solutions that correspond to the minimum-induced-drag solutions for the elliptic planform and linearly-tapered planforms with $0 \leq R_{T} \leq 1$. Black: Optimum Lift Distribution, Gray: Elliptic Lift Distribution.

Note that the elliptic planform gives results that closely match those obtained using a linearly-tapered planform with taper ratio just over $R_{T}=0.4$. This agrees with traditional intuition based on classical lifting-line theory [44,45]. However, it is important to note that whereas the elliptic planform is commonly considered the optimum planform shape, the elliptic planform does not minimize induced drag for this case. Instead, induced drag is minimized for both the fixed elliptic lift distribution and the optimum lift distribution at a taper ratio of $R_{T}=0$, which corresponds to a triangular wing. This agrees with the results shown in Figs. 2 and 7. Using the optimum lift distribution, the triangular planform allows a wingspan increase of $15.16 \%$ and an induced-drag reduction of $22.13 \%$ when compared to the rectangular planform. In contrast, using the optimum lift distribution, the elliptic planform gives a maximum reduction in induced drag of only $12.12 \%$ over the rectangular planform. Thus, for this example, the minimum induced drag is obtained with a linearly-tapered planform having a taper ratio of $R_{T}=0$, with $D_{i}=71.74617 \mathrm{lbf}$ at $B_{3}=-0.17193$ and $b=105.88820 \mathrm{ft}$. The optimum wing-structure weight for this solution is $W_{s}=3500 \mathrm{lbf}$. Induced-drag contours around the minimum-induced-drag solution are shown in Fig. 11 as a function of $B_{3}$ and wingspan.

Although the results presented in this section predict minimum induced drag at a taper ratio of $R_{T}=0$, it is important to remember that wings with low taper ratios have low Reynolds numbers near the wingtips, which often cause the wing to stall first in these regions. For most aircraft, this can create serious handling problems, especially during stall recovery. For this reason, wings with low taper ratios are seldom used in practice on aircraft. Nevertheless, the results presented here provide important insight into the aerodynamic and structural coupling in the stress-limited design of wings with elliptic and linearly-tapered planforms for minimum induced drag.

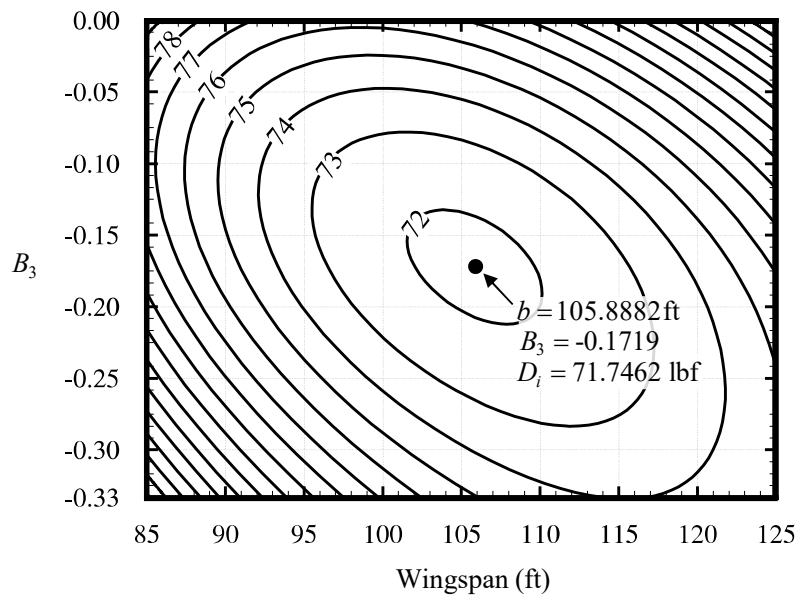

Fig. 11 Induced-drag contours around the minimum-induced drag solution for fixed net weight. 


\section{Conclusions}

From classical lifting-line theory, the induced drag on a wing in steady level flight is given by Eq. (2) and is a function of wingspan, wing weight, and lift distribution. For a given lift distribution, Eq. (2) is minimized by maximizing wingspan and/or minimizing wing weight. Increasing wingspan increases the required wing-structure weight, but certain non-elliptic lift distributions can alleviate bending moments near the wingtips, allowing an increase in wingspan with no increase in the wing-structure weight. However, any non-elliptic lift distribution incurs a penalty in induced drag. Thus, there exists an optimum wingspan and lift distribution that minimizes induced drag and depends on the tradeoff between wingspan, wing weight, and associated lift distribution.

In a 1933 paper, Prandtl noted that these optimum lift distributions correspond more closely to those produced by untwisted tapered wings than the elliptic lift distribution, giving tapered wings an advantage over nearly rectangular wings. However, Prandtl's mathematical development for finding the optimum wingspan and lift distribution that minimize induced drag was limited to rectangular wings. Here, we have relaxed many of the assumptions used in Prandtl's development so that it applies to non-rectangular wings. Closed-form solutions for the optimum wingspan and corresponding induced drag are given in Eqs. (27) and (28), respectively, for the elliptic planform with fixed gross weight, Eqs. (31) and (33) for the elliptic planform with fixed net weight, Eqs. (47) and (48) for linearly-tapered planforms with fixed gross weight, and Eqs. (51) and (53) for linearly-tapered planforms with fixed net weight. If the net weight is fixed and gross weight is allowed to vary, there also exists an optimum wing-structure weight that is one-half the net weight for both elliptic and linearly-tapered planforms. Thus, if the optimum wing-structure weight is used, the constraint of fixed gross weight and the constraint of fixed net weight are equivalent.

Figure 2 shows that for a linearly-tapered planform with a fixed lift distribution having $-1 / 3 \leq B_{3} \leq 0$ and $B_{n}=0$ for all $n \neq 3$, the induced drag is always minimized using a triangular planform having $R_{T}=0$. When compared to the rectangular planform, the triangular planform allows a wingspan increase of up to $15.04 \%$ and a reduction in induced drag of up to $24.44 \%$, depending on the lift distribution. When compared to the elliptic planform, the triangular planform gives a reduction in induced drag of up to $11.71 \%$. Results similar to these are typical for any fixed lift distribution; however, an absolute minimum in induced drag is not obtained unless the optimum lift distribution is also used.

In general, the optimum lift distribution that minimizes induced drag for a wing with an elliptic or linearly-tapered planform is an infinite Fourier series with coefficients $B_{n}$. In order to predict the optimum lift distribution, the infinite series must be truncated at some finite value of $n$ and the values of $B_{n}$ that minimize induced drag must be obtained numerically. For the planforms considered in this paper, the optimum values for $B_{n}$ are given in Table A3. These values depend on the planform shape and the design constraints. However, Fig. 4 shows that for the planforms and design constraints considered here, the general shape of the optimum lift distribution varies only slightly with planform shape. Figures 7 and 8 show that, when compared to the elliptic lift distribution, the optimum lift distribution can allow a wingspan increase of up to $7.63 \%$ and an induced-drag reduction of up to $5.94 \%$ at $R_{T}=0$. Thus, it has been shown that Prandtl's conclusions about tapered wings maintain their validity when the effects of planform on the wing-structure weight are taken into account.

It has also been shown that although the optimum lift distributions that minimize induced drag for wings with elliptic and linearly-tapered planforms depend on an infinite number of Fourier coefficients, for the planforms and design constraints considered here, a good approximation for the optimum lift distribution can be made by including only $B_{3}$ in the series defining the lift distribution. Figure 6 shows that the value of $B_{3}$ in the optimum lift distribution decreases as the taper ratio decreases.

The results presented in this paper provide valuable insight into the aerodynamic and structural coupling involved in the stress-limited design of wings with elliptic and linearly-tapered planforms for minimum induced drag. Although the results favor wings with low taper ratios, it should always be remembered that planforms with small chord values near the wingtips are seldom practical because they tend to stall at the wingtips and exhibit poor handling qualities, especially during stall recovery. Nevertheless, the results shown here may shed light on why many high-endurance birds have low-taper-ratio wings. It is also important to remember that we have only considered wings with the weight distribution given by Eqs. (5) and (6) and elliptic or linearly-tapered planforms with $0 \leq R_{T} \leq 1$. If any other weight distribution is used, the wing-structure weight, optimum wingspan, and minimum induced drag may need to be found using numerical methods. However, if the weight distribution given by Eqs. (5) and (6) is used, the methods presented in this paper can be repeated for any planform with a chord distribution that can be integrated in $z$. 


\section{Appendix}

As seen in Eq. (44), each of the coefficients, $C_{n}$, for a linearly-tapered wing is a nonlinear function of taper ratio and must be evaluated numerically. The result of evaluating Eq. (44) for taper ratios in the range $0 \leq R_{T} \leq 1$ is shown in Table A1. The composite Simpson's rule was used for all numerical integration. For convenience, each of the coefficients, $C_{n}$, was also fit, as a function of taper ratio, to a sixth-order polynomial of the form

$$
C_{n}=\sum_{m=0}^{6} a_{m} R_{T}^{m}
$$

where $a_{m}$ are the fit coefficients given in Table A2 for all odd $n \leq 29$ and taper ratios in the range $0.2 \leq R_{T} \leq 1.5$. Outside this range of taper ratios, the value of $C_{n}$ as a function of taper ratio cannot be accurately approximated using a polynomial of reasonably low order.

With the coefficients from Table A1, Eqs. (33) and (53) can be minimized using a numerical optimization framework with wingspan and the coefficients, $B_{n}$, as the design variables. Using the Broyden-Fletcher-GoldfarbShanno (BFGS) [40-43] method with the Fourier coefficients, $B_{n}$, for all odd $n$ in the range $3 \leq n \leq 29$ gives the optimum lift distributions shown in Fig. 4 and the optimum values for the Fourier coefficients, $B_{n}$, shown in Table A3. 
Table A1 $\boldsymbol{C}_{\boldsymbol{n}}$ coefficients for all odd $\boldsymbol{n} \leq \mathbf{2 9}$ for wings with linearly-tapered planforms having $0 \leq \boldsymbol{R}_{\boldsymbol{T}} \leq \mathbf{1}$.

\begin{tabular}{|c|c|c|c|c|c|c|c|c|c|c|c|}
\hline & $R_{T}=0.0$ & $R_{T}=0.1$ & $R_{T}=0.2$ & $R_{T}=0.3$ & $R_{T}=0.4$ & $R_{T}=0.5$ & $R_{T}=0.6$ & $R_{T}=0.7$ & $R_{T}=0.8$ & $R_{T}=0.9$ & $R_{T}=1.0$ \\
\hline$C_{1}$ & $2.7716 \times 10^{-1}$ & $2.6155 \times 10^{-1}$ & $2.4966 \times 10^{-1}$ & $2.3983 \times 10^{-1}$ & $2.3139 \times 10^{-1}$ & $2.2398 \times 10^{-1}$ & $2.1737 \times 10^{-1}$ & $2.1140 \times 10^{-1}$ & $2.0596 \times 10^{-1}$ & $2.0097 \times 10^{-1}$ & $1.9635 \times 10^{-1}$ \\
\hline$C_{3}$ & $1562 \times 10^{-1}$ & $2.8932 \times 10^{-1}$ & $2.7072 \times 10^{-1}$ & $2.5600 \times 10^{-1}$ & $2.4378 \times 10^{-1}$ & $2.3332 \times 10^{-1}$ & $2.2420 \times 10^{-1}$ & $2.1612 \times 10^{-1}$ & $2.0887 \times 10^{-1}$ & $2.0232 \times 10^{-1}$ & $1.9635 \times 10^{-1}$ \\
\hline$C_{5}$ & $4.3190 \times 10^{-2}$ & $2.8797 \times 10^{-2}$ & $2.0825 \times 10^{-2}$ & $1.5437 \times 10^{-2}$ & $1.1491 \times 10^{-2}$ & $8.4614 \times 10^{-3}$ & $6.0592 \times 10^{-3}$ & $4.1092 \times 10^{-3}$ & $2.4975 \times 10^{-3}$ & $1.1462 \times 10^{-3}$ & 0 \\
\hline$C_{7}$ & $7.6085 \times 10^{-3}$ & $2.1946 \times 10^{-3}$ & $5.1974 \times 10^{-4}$ & $-1.5985 \times 10^{-4}$ & $-4.2757 \times 10^{-4}$ & $-4.9724 \times 10^{-4}$ & $-4.6465 \times 10^{-4}$ & $-3.7786 \times 10^{-4}$ & $-2.6275 \times 10^{-4}$ & $-1.3397 \times 10^{-4}$ & 0 \\
\hline$C_{9}$ & $3.4235 \times 10^{-3}$ & $9.5812 \times 10^{-4}$ & $4.8609 \times 10^{-4}$ & $3.2466 \times 10^{-4}$ & $2.4850 \times 10^{-4}$ & $1.9910 \times 10^{-4}$ & & $1.1872 \times 10^{-4}$ & & & 0 \\
\hline$C_{11}$ & $1.1890 \times 10^{-3}$ & $5.1200 \times 10^{-6}$ & $-9.7040 \times 10^{-5}$ & $-1.0446 \times 10^{-4}$ & $-9.4190 \times 10^{-5}$ & $-7.9840 \times 10^{-5}$ & $-6.4390 \times 10^{-5}$ & $-4.8540 \times 10^{-5}$ & $-3.2470 \times 10^{-5}$ & $-1.6270 \times 10^{-5}$ & 0 \\
\hline$C_{13}$ & $7.5613 \times 10^{-4}$ & $1.0620 \times 10^{-4}$ & $6.6420 \times 10^{-5}$ & $5.5000 \times 10^{-5}$ & $4.6900 \times 10^{-5}$ & $3.9190 \times 10^{-5}$ & $3.1470 \times 10^{-5}$ & $2.3670 \times 10^{-5}$ & $1.5820 \times 10^{-5}$ & $7.9200 \times 10^{-6}$ & 0 \\
\hline$C_{15}$ & $3.3871 \times 10^{-4}$ & $-2.7780 \times 10^{-5}$ & $-3.3150 \times 10^{-5}$ & $-2.9780 \times 10^{-5}$ & $-2.5690 \times 10^{-5}$ & $-2.1480 \times 10^{-5}$ & $-1.7230 \times 10^{-5}$ & $-1.2950 \times 10^{-5}$ & $-8.6500 \times 10^{-6}$ & $-4.3300 \times 10^{-6}$ & 0 \\
\hline$C_{17}$ & $2.5479 \times 10^{-4}$ & $2.5700 \times 10^{-5}$ & $2.0420 \times 10^{-5}$ & $1.7800 \times 10^{-5}$ & $1.5300 \times 10^{-5}$ & $1.2780 \times 10^{-5}$ & $1.0240 \times 10^{-5}$ & $7.7000 \times 10^{-6}$ & $5.1400 \times 10^{-6}$ & $2.5700 \times 10^{-6}$ & 0 \\
\hline$C_{19}$ & $1.3071 \times 10^{-4}$ & $-1.3500 \times 10^{-5}$ & $-1.2810 \times 10^{-5}$ & $-1.1270 \times 10^{-5}$ & $-9.6800 \times 10^{-6}$ & $-8.0800 \times 10^{-6}$ & $-6.4800 \times 10^{-6}$ & $-4.8600 \times 10^{-6}$ & $-3.2500 \times 10^{-6}$ & $-1.6200 \times 10^{-6}$ & 0 \\
\hline$C_{21}$ & & & & $7.4800 \times 10^{-6}$ & $6.4300 \times 10^{-6}$ & $5.3600 \times 10^{-6}$ & $4.3000 \times 10^{-6}$ & $3.2300 \times 10^{-6}$ & $2.1500 \times 10^{-6}$ & $1.0800 \times 10^{-6}$ & 0 \\
\hline$C_{23}$ & $6.0660 \times 10^{-5}$ & $-6.5300 \times 10^{-6}$ & $-5.8900 \times 10^{-6}$ & $-5.1700 \times 10^{-6}$ & $-4.4400 \times 10^{-6}$ & $-3.7000 \times 10^{-6}$ & $-2.9600 \times 10^{-6}$ & $-2.2300 \times 10^{-6}$ & $-1.4800 \times 10^{-6}$ & $-7.4000 \times 10^{-7}$ & 0 \\
\hline$C_{25}$ & $5.3880 \times 10^{-5}$ & $4.7500 \times 10^{-6}$ & $4.2000 \times 10^{-6}$ & $3.6800 \times 10^{-6}$ & $3.1600 \times 10^{-6}$ & $2.6400 \times 10^{-6}$ & $2.1100 \times 10^{-6}$ & $1.5900 \times 10^{-6}$ & $1.0600 \times 10^{-6}$ & $5.3000 \times 10^{-7}$ & 0 \\
\hline$C_{27}$ & $3.1880 \times 10^{-5}$ & $-3.4500 \times 10^{-6}$ & $-3.0800 \times 10^{-6}$ & $-2.7000 \times 10^{-6}$ & $-2.3100 \times 10^{-6}$ & $-1.9300 \times 10^{-6}$ & $-1.5500 \times 10^{-6}$ & $-1.1600 \times 10^{-6}$ & $-7.7000 \times 10^{-7}$ & $-3.9000 \times 10^{-7}$ & 0 \\
\hline$C_{29}$ & $2.9690 \times 10^{-5}$ & $2.5900 \times 10^{-6}$ & $2.3100 \times 10^{-6}$ & $2.0200 \times 10^{-6}$ & $1.7300 \times 10^{-6}$ & $1.4500 \times 10^{-6}$ & $1.1600 \times 10^{-6}$ & $8.7000 \times 10^{-7}$ & $5.8000 \times 10^{-7}$ & $2.9000 \times 10^{-7}$ & 0 \\
\hline
\end{tabular}


Table A2 Fit coefficients in the polynomial approximation for $C_{n}$ as a function of taper ratio for wings with linearly-tapered planforms having $0.2 \leq R_{T} \leq 1.5$.

\begin{tabular}{lcccccccc}
\hline \hline & $a_{0}$ & $a_{1}$ & $a_{2}$ & $a_{3}$ & $a_{4}$ & $a_{5}$ & $a_{6}$ & $\mathrm{RMS}$ \\
\hline$C_{1}$ & $2.7264 \times 10^{-1}$ & $-1.3235 \times 10^{-1}$ & $8.8200 \times 10^{-2}$ & $-4.0101 \times 10^{-2}$ & $7.9669 \times 10^{-3}$ & 0 & 0 & $5.3456 \times 10^{-3}$ \\
$C_{3}$ & $3.0641 \times 10^{-1}$ & $-2.1072 \times 10^{-1}$ & $1.6649 \times 10^{-1}$ & $-8.3750 \times 10^{-2}$ & $1.7923 \times 10^{-2}$ & 0 & 0 & $7.9136 \times 10^{-3}$ \\
$C_{5}$ & $3.8614 \times 10^{-2}$ & $-1.2292 \times 10^{-1}$ & $2.1171 \times 10^{-1}$ & $-2.4417 \times 10^{-1}$ & $1.7341 \times 10^{-1}$ & $-6.7698 \times 10^{-2}$ & $1.1056 \times 10^{-2}$ & $2.3554 \times 10^{-3}$ \\
$C_{7}$ & $4.1527 \times 10^{-3}$ & $-3.0318 \times 10^{-2}$ & $7.8593 \times 10^{-2}$ & $-1.0626 \times 10^{-1}$ & $8.1457 \times 10^{-2}$ & $-3.3185 \times 10^{-2}$ & $5.5653 \times 10^{-3}$ & $1.7653 \times 10^{-3}$ \\
$C_{9}$ & $1.3151 \times 10^{-3}$ & $-7.0966 \times 10^{-3}$ & $1.9698 \times 10^{-2}$ & $-2.9850 \times 10^{-2}$ & $2.4629 \times 10^{-2}$ & $-1.0516 \times 10^{-2}$ & $1.8203 \times 10^{-3}$ & $1.0419 \times 10^{-3}$ \\
$C_{11}$ & $1.5970 \times 10^{-5}$ & $-1.1787 \times 10^{-3}$ & $4.0982 \times 10^{-3}$ & $-6.4574 \times 10^{-3}$ & $5.5203 \times 10^{-3}$ & $-2.4288 \times 10^{-3}$ & $4.3041 \times 10^{-4}$ & $5.8846 \times 10^{-4}$ \\
$C_{13}$ & $1.0667 \times 10^{-4}$ & $-3.1870 \times 10^{-4}$ & $7.8674 \times 10^{-4}$ & $-1.2858 \times 10^{-3}$ & $1.1205 \times 10^{-3}$ & $-4.9856 \times 10^{-4}$ & $8.9100 \times 10^{-5}$ & $2.8810 \times 10^{-4}$ \\
$C_{15}$ & $-4.1110 \times 10^{-5}$ & $3.6170 \times 10^{-5}$ & $7.4800 \times 10^{-6}$ & $-2.5400 \times 10^{-6}$ & 0 & 0 & 0 & $2.0678 \times 10^{-4}$ \\
$C_{17}$ & $2.5570 \times 10^{-5}$ & $-2.5570 \times 10^{-5}$ & 0 & 0 & 0 & 0 & 0 & $1.6191 \times 10^{-4}$ \\
$C_{19}$ & $-1.6170 \times 10^{-5}$ & $1.6170 \times 10^{-5}$ & 0 & 0 & 0 & 0 & 0 & $1.2791 \times 10^{-4}$ \\
$C_{21}$ & $1.0730 \times 10^{-5}$ & $-1.0730 \times 10^{-5}$ & 0 & 0 & 0 & 0 & 0 & $9.0310 \times 10^{-5}$ \\
$C_{23}$ & $-7.4000 \times 10^{-6}$ & $7.4000 \times 10^{-6}$ & 0 & 0 & 0 & 0 & 0 & $7.2520 \times 10^{-5}$ \\
$C_{25}$ & $5.2700 \times 10^{-6}$ & $-5.2700 \times 10^{-6}$ & 0 & 0 & 0 & 0 & 0 & $5.5860 \times 10^{-5}$ \\
$C_{27}$ & $-3.8600 \times 10^{-6}$ & $3.8600 \times 10^{-6}$ & 0 & 0 & 0 & 0 & 0 & $4.4150 \times 10^{-5}$ \\
$C_{29}$ & $2.8900 \times 10^{-6}$ & $-2.8900 \times 10^{-6}$ & 0 & 0 & 0 & 0 & 0 & $3.5500 \times 10^{-5}$ \\
\hline \hline
\end{tabular}


Table A3 Optimum $\boldsymbol{B}_{\boldsymbol{n}}$ coefficients for the elliptic planform and linearly-tapered planforms having $\mathbf{0} \leq \boldsymbol{R}_{\boldsymbol{T}} \leq \mathbf{1}$.

\begin{tabular}{|c|c|c|c|c|c|c|c|c|c|c|c|c|}
\hline & & & & & & & & $R_{T}=0.6$ & $R_{T}=0.7$ & & $R_{T}=0.9$ & \\
\hline$B_{3}$ & & & & & & & & & & & $-1.3709 \times 10^{-1}$ & \\
\hline$B_{5}$ & $-2.9064 \times 10^{-3}$ & & & & & & & & & & & \\
\hline$B_{7}$ & $-3.2293 \times 10^{-4}$ & 77004 & $2567 \times 10$ & -4 & & & & & & & & \\
\hline$B_{9}$ & & & & & & & & & & & & \\
\hline$B_{11}$ & & & & & & -5 & & -5 & $0^{-6}$ & 5.88 & & \\
\hline$B_{13}$ & & .505 & $.3697 \times 10^{-5}$ & -8.809 & & & & & $-3.5519 \times 10^{-6}$ & -2.42 & & \\
\hline & & & & & & & & & & & & \\
\hline$B_{17}$ & & 4 & $0^{-6}$ & -2.0 & & & & & -8.8 & & & \\
\hline & & & & & & & & & & & & \\
\hline$B_{21}$ & & -8.454 & $\times 10^{-7}$ & -7.014 & & & & & & & $10^{-7}$ & \\
\hline$B_{23}$ & & $-4.3099 \times 10^{-6}$ & $4.7602 \times 10^{-7}$ & $4.4285 \times 10^{-7}$ & $3.9864 \times 10^{-7}$ & & & & & & $\times 10^{-8}$ & -5.800 \\
\hline$B_{25}$ & & & $.1846 \times 10^{-7}$ & $-2.9050 \times 10^{-7}$ & $-2.6115 \times 10^{-7}$ & $-2.2987 \times 10^{-7}$ & $-1.9673 \times 10^{-7}$ & $-1.6023 \times 10^{-7}$ & $-1.2354 \times 10^{-7}$ & $\times 10^{-8}$ & $\times 10^{-8}$ & $0^{-10}$ \\
\hline$B_{27}$ & & $-1.9291 \times 10^{-6}$ & $2.1416 \times 10^{-7}$ & $1.9705 \times 10^{-7}$ & $1.7719 \times 10^{-7}$ & $1.5597 \times 10^{-7}$ & $1.3237 \times 10^{-7}$ & $1.0902 \times 10^{-7}$ & $8.3873 \times 10^{-8}$ & $5.3819 \times 10^{-8}$ & $2.9154 \times 10^{-8}$ & $-1.9700 \times 10^{-10}$ \\
\hline & $-5.3073 \times 10^{-8}$ & $-1.6725 \times 10^{-6}$ & $-1.5014 \times 10^{-7}$ & $-1.3694 \times 10^{-7}$ & $-1.2425 \times 10^{-7}$ & $-1.0813 \times 10^{-7}$ & $-9.3527 \times 10^{-8}$ & $-7.5954 \times 10^{-8}$ & $-5.4803 \times 10^{-8}$ & $-3.9496 \times 10^{-8}$ & $-1.9609 \times 10^{-8}$ & $5.1200 \times 10^{-10}$ \\
\hline
\end{tabular}




\section{Acknowledgements}

This material is partially based upon work supported by the National Aeronautics and Space Administration under Grant No. 80NSSC18K1696 issued through the Aeronautics Research Mission Directorate through the 2018 NASA Fellowship Activity, with Nhan Nguyen as the NASA Technical Advisor.

\section{References}

[1] Prandtl, L., "Tragflügel Theorie,” Nachricten von der Gesellschaft der Wissenschaften zu Güttingen, Ges-chäeftliche Mitteilungen, Klasse, 1918, pp. 451-477.

[2] Prandtl, L., "Applications of Modern Hydrodynamics to Aeronautics," NACA TR-116, June 1921.

[3] Phillips, W. F., and Hunsaker, D. F., "Designing Wing Twist or Planform Distributions for Specified Lift Distributions," Journal of Aircraft, Vol. 56, No. 2, 2019, pp. 847-849. (doi:10.2514/1.C035206)

[4] Phillips, W. F., "Lifting-Line Analysis for Twisted Wings and Washout-Optimized Wings," Journal of Aircraft, Vol. 41, No. 1, 2004, pp. 128-136. (doi:10.2514/1.262)

[5] Phillips, W. F., Fugal, S. R., and Spall, R. E., "Minimizing Induced Drag with Wing Twist, Computational-Fluid-Dynamics Validation," Journal of Aircraft, Vol. 43, No. 2, 2006, pp. 437-444. (doi:10.2514/1.15089)

[6] Gallay, S., and Laurendeau, E., "Preliminary-Design Aerodynamic Model for Complex Configurations Using Lifting-Line Coupling Algorithm," Journal of Aircraft, Vol. 53, No. 4, 2016, pp. 1145-1159. (doi:10.2514/1.C033460)

[7] Phillips, W. F., and Hunsaker, D. F., "Lifting-Line Predictions for Induced Drag and Lift in Ground Effect," Journal of Aircraft, Vol. 50, No. 4, 2013, pp. 1226-1233. (doi:10.2514/1.C032152)

[8] Wickenheiser, A., and Garcia, E., "Aerodynamic Modeling of Morphing Wings Using an Extended Lifting-Line Analysis," Journal of Aircraft, Vol. 44, No. 1, 2007, pp. 10-16. (doi:10.2514/1.18323)

[9] Phillips, W. F., and Snyder, D. O., "Modern Adaptation of Prandtl's Classic Lifting-Line Theory," Journal of Aircraft, Vol. 37, No. 4, 2000, pp. 662-670. (doi:10.2514/2.2649)

[10] Rasmussen, M. L., and Smith, D. E., "Lifting-Line Theory for Arbitrarily Shaped Wings," Journal of Aircraft, Vol. 36, No. 2, 1999, pp. 340-348. (doi:10.2514/2.2463)

[11] Bera, R. K., "Some remarks on the solution of the lifting line equation," Journal of Aircraft, Vol. 11, No. 10, 1974, pp. 647648. (doi:10.2514/3.44397)

[12] Phillips, W. F., Hunsaker, D. F., and Joo, J. J., "Minimizing Induced Drag with Lift Distribution and Wingspan,” Journal of Aircraft, Vol. 56, No. 2, 2019 pp. 431-441. (doi: 10.2514/1.C035027)

[13] McCormick, B. W., Aerodynamics, Aeronautics, and Flight Mechanics, 1st ed., Wiley, New York, 1979

[14] Prandtl, L., "Über Tragflügel kleinsten induzierten Widerstandes," Zeitschrift für Flugtechnik und Motorluftschiffahrt, Vol. 24, No. 11, 1933, pp. 305-306.

[15] Hunsaker, D. F., "Ludwig Prandtl's 1933 Paper Concerning wings for Minimum Induced Drag, Translation and Commentary," AIAA SciTech 2020 Forum, Orlando, Florida, 6-10 January 2020.

[16] Lundry, J. L., "Minimum Swept-Wing Induced Drag with Constraints on Lift and Pitching Moment," Journal of Aircraft, Vol. 4, 1967, pp. 73-74. (doi:10.2514/3.43797)

[17] Lissaman, P. B. S., and Lundry, J. L., “A Numerical Solution for the Minimum Induced Drag of Nonplanar Wings,” Journal of Aircraft, Vol. 5, 1968, pp. 17-21. (doi:10.2514/3.43901)

[18] Ashenberg, J., and Weihsradius, D., "Minimum Induced Drag of Wings with Curved Planform," Journal of Aircraft, Vol. 21, 1984, pp. 89-91. (doi:10.2514/3.56733)

[19] Rokhsaz, K., "Effect of Viscous Drag on Optimum Spanwise Lift Distribution," Journal of Aircraft, Vol. 30, 1993, pp. 152154. (doi:10.2514/3.46328)

[20] Demasi, L., "Induced Drag Minimization: A Variational Approach Using the Acceleration Potential," Journal of Aircraft, Vol. 43, 2006, pp. 669-680. (doi:10.2514/1.15982)

[21] Demasi, L., "Erratum on Induced Drag Minimization: A Variational Approach Using the Acceleration Potential," Journal of Aircraft, Vol. 43, 2006, p. 1247. (doi:10.2514/1.26648)

[22] Demasi, L., "Investigation on the Conditions of Minimum Induced Drag of Closed Wing Systems and C-Wings," Journal of Aircraft, Vol. 44, 2007, pp. 81-99. (doi:10.2514/1.21884)

[23] Demasi, L., Monegato, G., and Cavallaro, R., "Minimum Induced Drag Theorems for Multiwing Systems," AIAA Journal, Vol. 55, 2017, pp. 3266-3287. (doi:10.2514/1.J055652)

[24] Demasi, L., Dipace, A., Monegato, G., and Cavallaro, R., "Invariant Formulation for the Minimum Induced Drag Conditions of Nonplanar Wing Systems,” AIAA Journal, Vol. 52, 2014, pp. 2223-2240. (doi:10.2514/1.J052837) 
[25] Grossman, B., Gurdal, Z., Strauch, G. J., Eppard, W. M., and Haftka, R. T., "Integrated Aerodynamic/Structural Design of a Sailplane Wing," Journal of Aircraft, Vol. 25, No. 9, 1988, pp. 855-860. (doi: 10.2514/3.45670)

[26] Craig, A. P., and McLean, D. J., "Spanload Optimization for Strength Designed Lifting Surfaces," AIAA 88-2512, 6th Applied Aerodynamics Conference, Williamsburg, Virginia, 5-8 June 1988.

[27] Iglesias, S., and Mason, W. H., "Optimum Spanloads Incorporating Wing Structural Weight,” AIAA 2001-5234, 1st Aircraft, Technology Integration, and Operations Forum, Los Angeles, California, 16-18 October 2001.

[28] Wroblewski, G. E., and Ansell, P. J., "Prediction and Experimental Evaluation of Planar Wing Spanloads for Minimum Drag," Journal of Aircraft, Vol. 54, 2017, pp. 1664-1674. (doi:10.2514/1.C034156)

[29] Gopalarathnam, A., and Norris, R. K., "Ideal Lift Distributions and Flap Angles for Adaptive Wings," Journal of Aircraft, Vol. 46, No. 2, 2009, pp. 562-571. (doi: 10.2514/1.38713)

[30] McGeer, T., "Wing Design for Minimum Drag with Practical Constraints," Journal of Aircraft, Vol. 21, 1984, pp. 879-886. (doi:10.2514/3.45058)

[31] Pate, D. J., and German, B. J., "Lift Distributions for Minimum Induced Drag with Generalized Bending Moment Constraints," Journal of Aircraft, Vol. 50, 2013, pp. 936-946. (doi:10.2514/1.C032074)

[32] Jones, R. T., “The Spanwise Distribution of Lift for Minimum Induced Drag of Wings Having a Given Lift and a Given Bending Moment," NACA TR-2249, December 1950.

[33] DeYoung, J., "Minimization Theory of Induced Drag Subject to Constraint Conditions," NASA CR-3140, June 1979.

[34] Jones, R. T., and Lasinski, T. A., "Effect of Winglets on the Induced Drag of Ideal Wing Shapes," NASA TM-81230, September 1980 .

[35] Klein, A., and Viswanathan, S. P., "Minimum Induced Drag of Wings with Given Lift and Root-Bending Moment," Zeitschrift fur Angewandte Mathematik und Physik, Vol. 24, 1973, pp. 886-892.

[36] Klein, A., and Viswanathan, S. P., "Approximate Solution for Minimum Induced Drag of Wings with Given Structural Weight," Journal of Aircraft, Vol. 12, No. 2, 1975, pp. 124-126. (doi:10.2514/3.44425)

[37] Löbert, G., "Spanwise Lift Distribution of Forward- and Aft-Swept Wings in Comparison to the Optimum Distribution Form," Journal of Aircraft, Vol. 18, No. 4, 1981, pp. 496-498. (doi: 10.2514/3.44717)

[38] Phillips, W. F., Hunsaker, D. F., and Taylor, J. D., "Minimizing Induced Drag with Weight Distribution, Lift Distribution, Wingspan, and Wing-Structure Weight," AIAA 2019-3349, AIAA Aviation 2019 Forum, Dallas, Texas, 17-21 June 2019. (doi: 10.2514/6.2019-3349)

[40] Broyden, C., "The convergence of a Class of Double-Rank Minimization Algorithms," Journal of the Institute of Mathematics and its Applications, Vol. 6, 1970 pp. 76-90. (doi: 10.1093/imamat/6.1.76)

[41] Fletcher, R., "A New Approach to Variable Metric Algorithms," Computer Journal, Vol. 13, No. 3, 1970, pp. 317-322. (doi: 10.1093/comjnl/13.3.317)

[42] Goldfarb, D., “A Family of Variable Metric Updates Derived by Variational Means," Mathematics of Computation, Vol. 24, No. 109, 1970, pp. 23-26. (doi: 10.1090/S0025-5718-1970-0258249-6)

[43] Shanno, D., "Conditioning of Quasi-Newton Methods for Function Minimization," Mathematics of Computation, Vol. 24, No. 111, 1970, pp. 647-656. (doi: 10.1090/S0025-5718-1970-0274029-X)

[44] Glauert, H., The Elements of Aerofoil and Airscrew Theory, Cambridge University Press, London, 1926.

[45] Phillips, W. F., "Incompressible Flow over Finite Wings," Mechanics of Flight, 2nd ed., Wiley, Hoboken, NJ, 2010, pp. 4694. 